\title{
Experimental investigation of a rotor blade tip vortex pair
}

\author{
Dominic Schröder ${ }^{1}$ (D) . Jorge Aguilar-Cabello ${ }^{2,3} \cdot$ Thomas Leweke $^{2} \cdot$ Ralf Hörnschemeyer $^{1} \cdot$ Eike Stumpf $^{1}$
}

Received: 1 April 2021 / Revised: 20 September 2021 / Accepted: 1 October 2021 / Published online: 20 October 2021

(C) The Author(s) 2021

\begin{abstract}
This paper presents the results of an experimental study of two closely spaced vortices generated by a rotating blade with a modified tip geometry. The experiments are carried out in two water channel facilities and involve a generic one-bladed rotor operating in a regime near hover. It is equipped with a parametric fin placed perpendicular to the pressure surface near the tip, which generates a co-rotating vortex pair having a helical geometry. Based on previous results obtained with a fixed wing, a series of small-scale experiments is first carried out, to validate the method of vortex pair generation also for a rotating blade, and to obtain a qualitative overview of its evolution going downstream. A more detailed quantitative study is then performed in a larger facility at three times the initial scale. By varying the fin parameters, it was possible to obtain a configuration in which the two vortices have almost the same circulation. In both experiments, the vortex pair is found to merge into a single helical wake vortex within one blade rotation. Particle image velocimetry measurements show that the resulting vortex has a significantly larger core radius than the single tip vortex from a blade without fin. This finding may have relevance in the context of blade-vortex interactions, where noise generation and fatigue from fluid-structure interactions depend strongly on the vortex core size.
\end{abstract}

Keywords Rotor wake $\cdot$ Blade tip vortex $\cdot$ Vortex interactions $\cdot$ Vortex merging

$\begin{array}{ll}\text { List of symbols } \\ a & \text { Vortex core radius [cm] } \\ a_{\max } & \text { Radius of maximum swirl velocity [cm] } \\ B & \text { Span of the fixed wing [cm] } \\ b & \text { Vortex separation distance [cm] } \\ c & \text { Chord [cm] } \\ f & \text { Rotation frequency [Hz] } \\ R & \text { Rotor radius [cm] } \\ R e & \text { Reynolds number, } 2 \pi R f c / v[-] \\ r & \text { Radial coordinate [cm] } \\ t & \text { Time [s] } \\ U & \text { Free-stream velocity [cm/s] } \\ v & \text { Tangential (swirl) velocity [cm/s] } \\ x & \text { Axial coordinate [cm] } \\ y & \text { Radial coordinate }[\mathrm{cm}] \\ z & \text { Spanwise coordinate [cm] }\end{array}$

Dominic Schröder

schroeder@ilr.rwth-aachen.de

1 Institute of Aerospace Systems (ILR), RWTH Aachen University, 52062 Aachen, Germany

2 IRPHE, CNRS, Aix-Marseille Université, Centrale Marseille, 13384 Marseille, France

3 Escuela de Ingenierías Industriales, Universidad de Málaga, 29071 Málaga, Spain

$\begin{array}{ll}\alpha & \text { Effective angle of attack }\left[{ }^{\circ}\right] \\ \alpha_{\mathrm{f}} & \text { Geometric fin angle }\left[{ }^{\circ}\right] \\ \Gamma & \text { Vortex circulation }\left[\mathrm{cm}^{2} / \mathrm{s}\right] \\ \Gamma_{\mathrm{b}} & \text { Bound circulation }\left[\mathrm{cm}^{2} / \mathrm{s}\right] \\ \lambda & \text { Tip speed ratio, } 2 \pi R f / U[-] \\ \nu & \text { Kinematic viscosity }\left[\mathrm{cm}^{2} / \mathrm{s}\right] \\ \Omega & \text { Angular vortex pair velocity }[\mathrm{rad} / \mathrm{s}] \\ \omega & \text { Vorticity [1/s] } \\ \phi & \text { Phase angle of the blade (wake age) }\left[{ }^{\circ}\right] \\ \varphi & \text { Azimuthal coordinate of local polar grid }\left[{ }^{\circ}\right] \\ \rho & \text { Radial coordinate of local polar grid }[\mathrm{cm}] \\ \theta & \text { Local blade twist }\left[{ }^{\circ}\right]\end{array}$

\section{Abbreviations}

BVI Blade-vortex interaction

PIV Particle image velocimetry

FOV Field of view

\begin{tabular}{ll}
\multicolumn{2}{l}{ Subscripts } \\
0 & Unmodified blade \\
$\mathrm{t}$ & Blade tip \\
$\mathrm{f}$ & Fin \\
tot & Total \\
$\max$ & Maximum
\end{tabular}




\section{Introduction}

\subsection{Background and objective}

The flow around rotors is characterised by the existence of helical vortices in its wake. Rotating blades, like fixed wings, cause the formation of concentrated vortices at their tips, due to the pressure equalisation in the blade tip region, and the combination of axial velocity (free-stream and/or rotor-induced) and rotational motion of the blade leads to their characteristic helical shape. Helical vortex wakes and their dynamics are of significant importance for many practical issues. Regarding helicopters, the helical tip vortices and their interaction with a following blade (blade-vortex-interaction, BVI) can generate unwanted noise and cause vibrations of the structure in certain flight regimes [1]. The fatigue load incurred by a wind turbine, as well as its performance, can be affected adversely by the wake of another turbine placed upstream [2]. In both cases, the strength of the local velocity gradients, with the corresponding rapid changes in local rotor blade air loads, is a crucial parameter. The circulation and core radius of the tip vortices, which both influence these gradients, are therefore vital elements affecting the strength of the interactions between these vortices and the following blades [3].

Minimising the negative effects of BVI has been targeted in several studies over the past years, generating a variety of both active and passive solutions. Concepts involving active rotor control systems are presented in the review by $\mathrm{Yu}$ et al. [4]. The governing approach of passive systems is to influence the vortex parameters, to decrease the interaction with the following blade. Various concepts for blade tip geometries and their influence on the vortices have been investigated, aiming at splitting or diffusing the tip vortex [5]. One operating principle of those tip shapes is to create multiple vortices that interact and merge, resulting in a larger final vortex with lower gradients, which is beneficial for mitigating BVI. The present study is also based on this idea: we here consider splitting the blade tip vortex into two separate co-rotating vortices.

The objective of this work is to gain a deeper physical understanding of the interaction phenomena occurring in such a closely spaced helical vortex system, which may involve instabilities of the individual vortices and of the vortex system, as well as merging of the vortex cores. A special blade geometry is utilised to generate a tip vortex pair of almost equal circulations. The concept of generating a second vortex by a perpendicular fin had been successfully tested and analysed in previous fixed wing experiments [6]. Building on these results, a rotor blade with a similar outer circulation distribution as the fixed wing is designed and investigated first in a small-scale experiment. To allow for a detailed quantitative analysis of the generated vortex system, including the core structure, and the effect on the rotor far wake, a second larger-scale experiment is built in a different facility. The spatial and temporal evolution of both vortices, as well as their merging process is then analysed in detail using dye visualisation and particle image velocimetry.

The paper is organised as follows. After recalling certain theoretical results concerning relevant vortex interaction mechanisms for the current configuration in Sect. 2, we discuss in Sect. 3 the design of the single-bladed rotor used in this work and present the two experimental facilities in which it was tested. Section 4 contains the results from these measurements, which first validate the concept of the new tip geometry for a rotating wing, and then show details of the helical tip vortex evolution and merging. Conclusions are given in Sect. 5.

\section{Theoretical aspects}

\subsection{Instabilities of vortex systems}

The physics of vortex systems has been investigated in numerous studies. A review regarding the two-dimension-al dynamics and the three-dimensional instabilities of co- and counter-rotating straight vortex pairs is presented in Ref. [7]. The instability mechanisms can be divided into two groups (despite the geometrical differences, this distinction can also be made for helical vortex systems [8]). The first involves a local displacement of the whole vortex with minimal effects on the internal structure. Considering the associated wavelength of the perturbations in comparison to the vortex core diameter, the interactions are described as long-wavelength instabilities. Widnall [9] investigated the stability of a single helical vortex filament using self-induced velocities governed by the Biot-Savart law. An extension of this work to multiple interlaced helical vortices was made in the theoretical analysis by Gupta and Loewy [10]. The limitation of these studies is that the Biot-Savart integral for the flow induced by a helical filament does not have a closed form. An analytical solution for the velocity field induced by a helical filament was presented by Kawada [11] and Hardin [12]. Okulov [13] used these results and provided the analytical solution of the generalized stability problem for multiple helical vortices. An experimental study focussing on the long-wave instability of helical vortices was presented by Quaranta et al. [14, 15], involving the investigation of a single vortex and a configuration consisting of two interlaced helical vortices.

A second type of instability arises inside the vortex core, with perturbation wavelengths of the order of the core 
diameter. Moore and Saffman [16] identified the resonance of Kelvin modes due to an outer strain field as the origin of the instability. The present term used to describe this phenomenon, the elliptic instability, was introduced by Kerswell [17]. Leweke and Williamson [18] provided experimental evidence of the elliptic instability in straight counter-rotating vortex pairs without axial core flow. Meunier and Leweke [19] extended this work by studying the effect of threedimensional instabilities on the vortex merging process of co-rotating vortices. The effect of an axial core flow was investigated numerically and experimentally by Roy et al. $[20,21]$.

In helical vortex systems, Blanco-Rodríguez et al. [22] identified curvature and torsion as additional sources of short-wavelength instabilities responsible for internal core deformations. In a later study, Blanco-Rodríguez and Le Dizès [23] proved analytically that the curvature adds a contribution to the elliptic instability growth rate. Besides the elliptic instability, another short-wave phenomenon is found in vortices with a curved axis, the curvature instability. A comprehensive analysis of this instability phenomenon is presented by Fukumoto and Hattori [24] for a vortex ring. They extended their work to helical vortex systems, also including axial core flow [25, 26]. Blanco-Rodríguez and Le Dizès [27] presented an analytical investigation of the curvature instability in a helical vortex using the Batchelor model. A comparison between both short-wavelength instability mechanisms is provided by Hattori et al. [28], deducing that the vortex swirl parameter has a crucial influence. Experimental work regarding short-wavelength instabilities in helical vortices can be found in Ref. [8]. It is shown that the interaction between short-wave instabilities and the longwave deformation modes leads to a rapid disruption of the concentrated helical vortices, similar to the observations made for straight vortex pairs [18].

All the above phenomena are potentially relevant for the helical vortex pair configuration considered in the present study.

\subsection{Vortex merging}

In addition to mutual interaction phenomena, two like-signed vortices in a viscous flow eventually merge to form a single vortex. Vortex merging and the underlying physics have been the topic of numerous studies in the past, e.g. in the context of decaying turbulence, where it can lead to larger structures. The merging process affects the vortex characteristics between the two separate initial vortices and the final merged vortex. The vortex core radius $a_{\max }$, defined as distance from the vortex centre where the maximum swirl velocity occurs, is increased, whereas both the peak vorticity in the core region and the maximum tangential velocities are decreased [29]. Leweke et al. [7] provide an analysis of the two-dimensional dynamics of the merging process, showing that two co-rotating vortices of circulations $\Gamma_{1}$ and $\Gamma_{2}$ and separation distance $b$ rotate around each other at an angular velocity $\Omega=\left(\Gamma_{1}+\Gamma_{2}\right) /\left(2 \pi b^{2}\right)$. The relevant parameter for the onset of vortex merging is given by the ratio between core radius and separation distance. For an equivalent Gaussian core radius, given by $a \approx a_{\max } / 1.12$, it was shown that the critical ratio indicating the beginning of the merging procedure is approximately 0.24 . The initial characteristics of the vortex system have an influence on the properties of the final merged vortex. Experimental dye visualisations and numerical results for the two-dimensional evolution are provided by Meunier et al. [29]. They showed that the final core radius is proportional to the initial vortex separation distance and exhibits maximum values for circulation ratios $\Gamma_{1} / \Gamma_{2} \approx 1$. Consequently, the objective of the modified blade design in our study is the formation of two vortices of nearly equal strength.

Detailed studies of the physics of vortex merging involving experimental, numerical and theoretical methods are presented in Refs. [30-33]. In laminar flows, the vortex core radius grows by viscous diffusion, which induces only moderate growth rates. The evolution of the core radius as function of time $t$ in a fluid of kinematic viscosity $v$ is given by

$a=\sqrt{a^{2}(t=0)+4 v t}$.

Merging is strongly affected by the presence of short-wavelength instabilities. Meunier et al. [19,31] pointed out that the criterion for onset and the characteristic parameters of the final merged vortex are modified by the instabilities. The critical ratio $a / b$ initialising the process for threedimensional merging was found to be smaller than the corresponding value for the two-dimensional case. In addition, the instability influence leads to a larger core size than in the absence of instability, and to a greater decrease of the maximum tangential velocity. Meunier et al. [19] trace this observation to the large decrease of the maximum vorticity, which is almost unmodified in the two-dimensional case. The development of the instabilities depends on the initial parameters of the vortex system. The growth rate is proportional to $b^{-2}$, indicating that a rapid evolution would require a small value of $b$, which is contra-productive regarding the dependency of the final core size on $b$. These findings suggest the existence of an optimal separation distance for the initial vortex system, concerning the objective of a rapid merging process before the encounter with a following blade, at least when considering the short-wave instability mechanism. 


\section{Experiments}

\subsection{Blade design}

The concept of splitting the blade tip vortex of a lifting surface into two separate vortices using a fin had previously been tested experimentally on a rectangular fixed wing with a NACA 0012 cross-section profile and an aspect ratio $B /(2 c)=2.9$, where $B$ is the (full) span and $c$ the chord [6]. An extended parameter study lead to the identification of promising configurations producing vortex pairs of similar circulations. In order to use the findings of this initial validation for the case of a rotating wing, one has to take into account the differences in the relative flow conditions along the blade in these two configurations. The fixed wing is subject to a constant freestream velocity, whereas the apparent velocity due to the rotation of the blade varies linearly in the radial (spanwise) direction. This leads to qualitatively different distributions of bound circulation along the blades. For a fixed wing of constant chord, this circulation is maximum at the root and decreases, first gradually and then more rapidly, towards the tip. For a constant-chord rotating blade, it increases from zero at the hub to reach a maximum near the tip, before a sharp drop back to zero.

Figure 1 shows the spanwise/radial distributions of bound circulation, as well as the corresponding effective angles of attack, for the fixed wing of Ref. [6] and two rotating blades designed for the present experiments. They were obtained by employing lifting-line models of these wings, in which the trailing vorticity in the wake is represented by straight line vortices (for the fixed wing, see e.g. [34]) or by helical vortex filaments (for rotating wings, see [35]). These models use as inputs the chord and geometrical twist distributions (see Fig. 2), as well as the lift curve data for the given blade cross-sectional shape, and the rotation frequency $f$ and free-stream velocity $U$. The rotating blades in this study have a constant chord, and their twist was designed in a way to produce distributions of bound circulation and angle of attack that are similar to those of the previously employed fixed wing, at least in the tip region. It can then be expected that the considered modification of the tip geometry used to split the vortex will perform in a similar way in the two configurations. In practice, a first design stage was carried out using a procedure based on the Blade-Element Momentum Theory [36, 37], aiming for an effective angle of attack comparable to the fixed-wing case for a single rotating blade of the same aspect ratio. In a second stage, the obtained twist distribution was optimised by trial and error to produce the desired circulation distribution. Figure 1 shows that a satisfactory match between the fixed and rotating wings
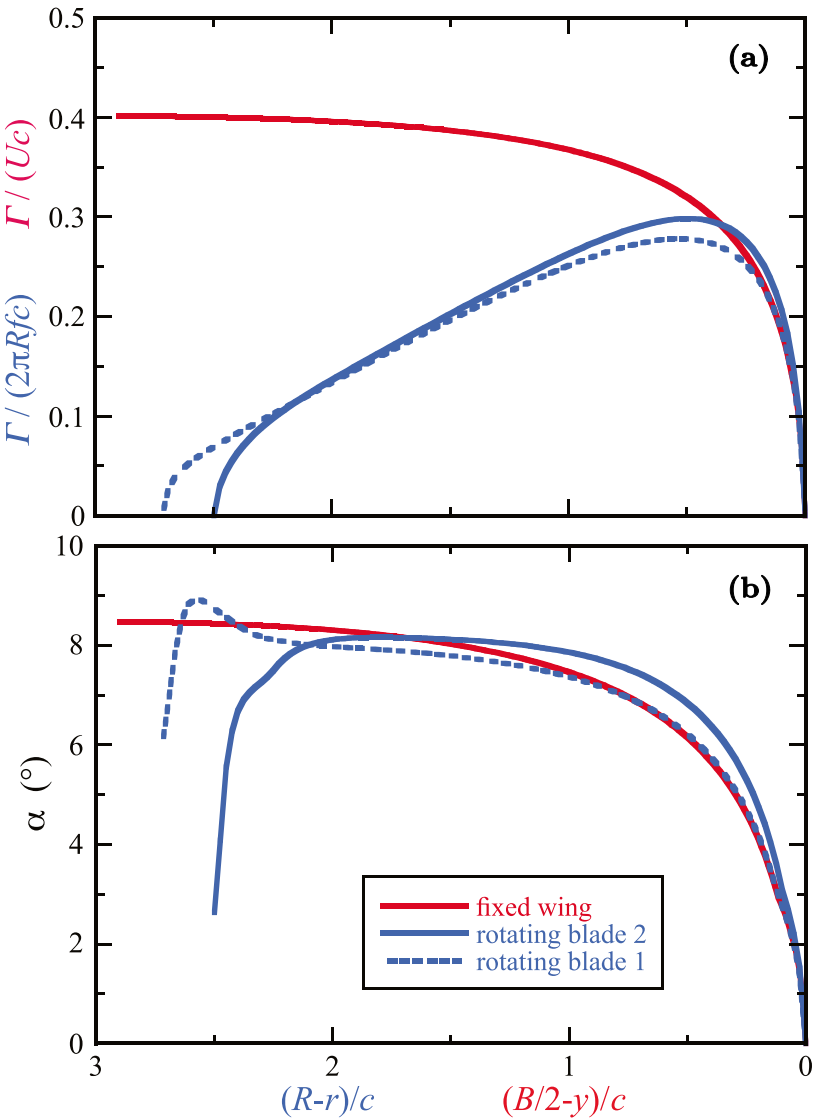

Fig. 1 Distributions of bound circulation (a) and effective angle of attack (b) along the fixed and rotating blades used in this study, plotted as function of the non-dimensional distance from the blade tip. Predictions based on lifting-line models with trailing vortex filaments

can be obtained in a spanwise interval of about $50 \%$ of the chord from the tip. This largely covers the region where the additional fin was placed.

With respect to previous work on lifting rotors (helicopters, drones), the initial reference condition for operation was hover. Since the measurements were carried out in water channels with test sections of finite size, it was found necessary to add a certain minimum free-stream velocity, leading to finite values of the tip speed ratio $\lambda$, to obtain a stable asymptotic flow and avoid large-scale unsteady recirculations. The final blade design took this into account. The parameters of the blades used in the two experiments described below, as well as the reference flow conditions, are listed in Table 1 and compared to those of the fixed wing in Ref. [6]. Both have an aspect ratio $R / c=3$, a cross-section shape given by a NACA 0012 profile and a rounded tip. The Reynolds number based on tip speed and chord was around $10^{5}$. The final twist distributions are shown in Fig. 2; they correspond to the result from the design procedure described above in the outer $75 \%$ of the blade. Near the rotor axis, adaptations were necessary, to fix the blade to the hub. 


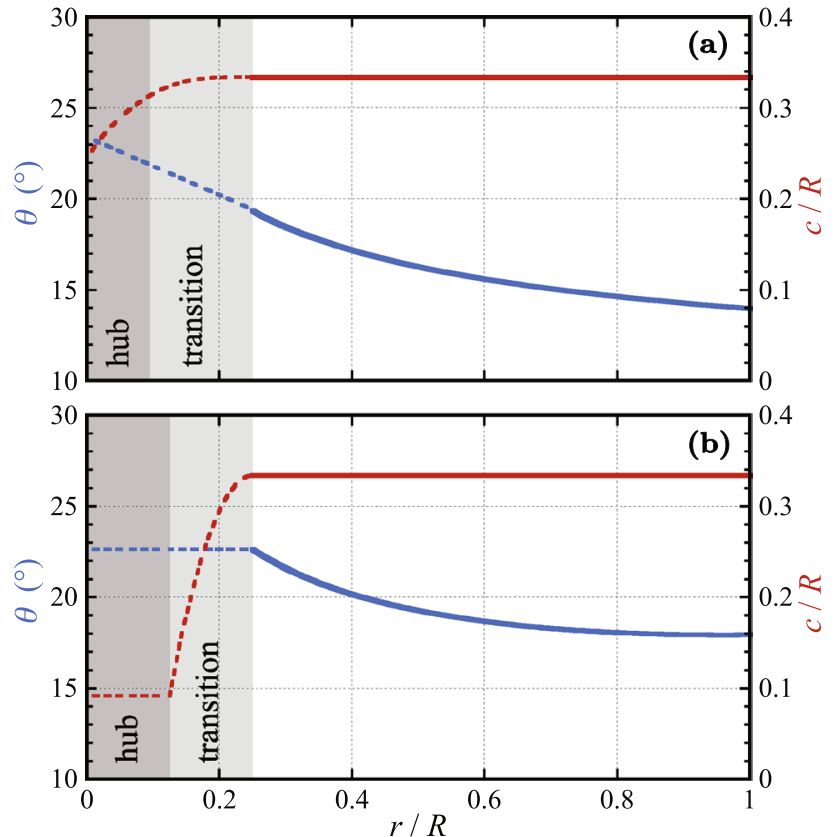

Fig. 2 Radial twist and chord distributions of the IRPHE (a) and ILR (b) blades

Table 1 Parameters of the base configurations used in the two experiments (IRPHE: small-scale; ILR: larger-scale), compared to the fixedwing case of Ref. [6]

\begin{tabular}{llll}
\hline & IRPHE & ILR & Fixed wing \\
\hline$R[\mathrm{~cm}]$ & 8 & 24 & $B / 2=29$ \\
$c[\mathrm{~cm}]$ & 2.67 & 8 & 10 \\
Aspect ratio & 3 & 3 & 2.9 \\
$U[\mathrm{~cm} / \mathrm{s}]$ & 5.0 & 11.5 & 100 \\
$f[\mathrm{~Hz}]$ & 6 & 1 & \\
$R e\left[10^{5}\right]$ & 0.8 & 1.2 & 1.0 \\
$\lambda$ & 60 & 13 & \\
$\mathrm{Hub} \varnothing[\mathrm{cm}]$ & 1.5 & 6 & \\
\hline
\end{tabular}

In all cases, the cross section has a NACA 0012 profile and the tip is rounded

The overall blade geometries can be seen in Fig. 3, which also shows the fin used to generate a second vortex and the various parameters defining its size, position and orientation, with respect to the blade tip. The fin consists of a short rectangular airfoil, which like the main blade has a NACA 0012 profile of constant chord and a rounded tip. As determined in [6], it works most efficiently when placed on the pressure side of the blade.

The smaller-scale validation experiment was carried out at the IRPHE institute, whereas the larger-scale tests (3 times bigger), allowing for a better resolution of the vortex core structure, were conducted at ILR. In the following, the two experiments are referred to by the respective
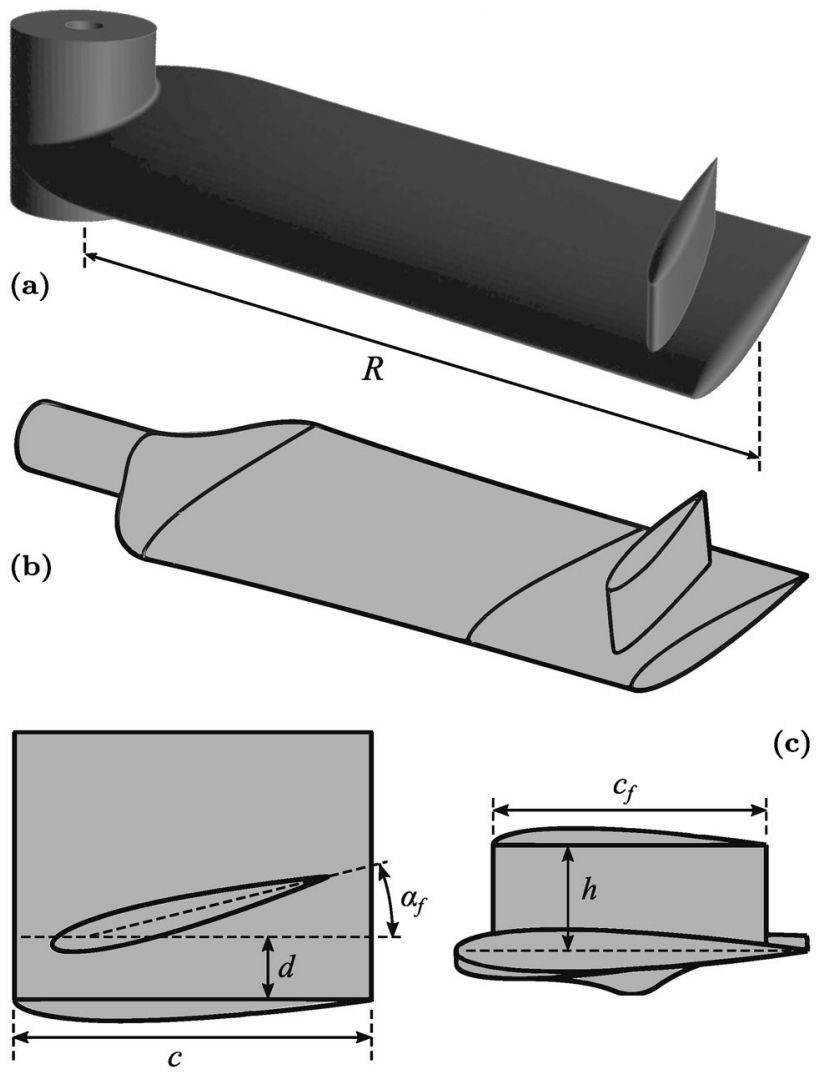

(c)

Fig. 3 Overall blade geometry for the IRPHE (a) and ILR (b) experiments. c Tip geometry and fin parameters

institute acronyms. In the IRPHE experiment, the fin configuration was varied by manufacturing a complete blade, including fin and hub, through rapid prototyping (high-resolution 3D printing), using a hard resin, for each parameter combination. For the larger set-up of the ILR experiment, only the outer $25 \%$ of the blade were replaced as a whole, also through $3 \mathrm{D}$ printing, for each new fin configuration.

\subsection{Facilities and methods}

The two experimental facilities used in this work are shown schematically in Fig. 4. It was decided to perform both experiments in water, since this provides favourable conditions for reliable quantitative investigations of vortex characteristics using tracer-based optical measurement techniques, such as Particle Image Velocimetry (PIV). In comparison to air, the balance between centrifugal and pressure forces on particles in the vortex cores is much easier to obtain, which ensures a suitable seeding density in the core region and consequently improves the accuracy of the PIV results there. Core seeding was monitored in both experimental set-ups and found sufficient for the present analysis. 

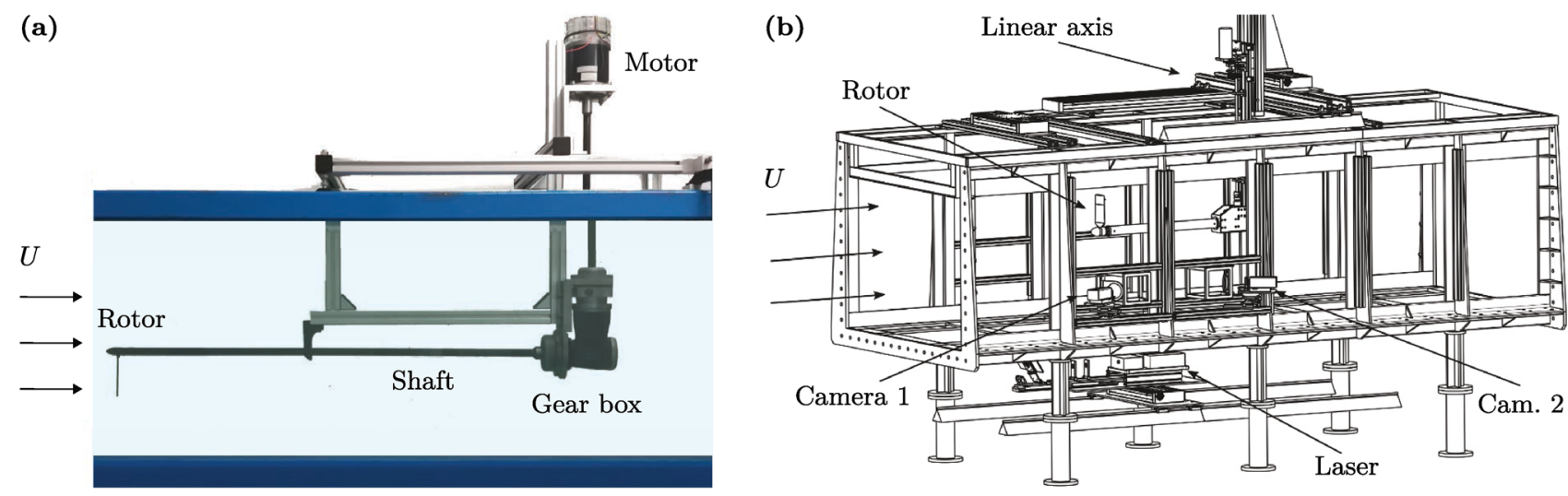

Fig. 4 Schematics of the two experimental set-ups (water channel test sections). a IRPHE experiment; b ILR experiment

\subsubsection{IRPHE experiment}

The small-scale validation experiment at IRPHE was carried out in a hydrodynamic channel with a recirculating freesurface water flow. The dimensions of the test section are $38 \mathrm{~cm} \times 50 \mathrm{~cm} \times 150 \mathrm{~cm}$. The rotor was mounted on a 1.5 $\mathrm{cm}$ diameter carbon shaft driven by a stepper motor placed outside the test section through a gear box. The rotor wake and tip vortices were characterised by velocity and vorticity fields obtained from two-component PIV measurements. A CCD camera (Roper Redlake) with a resolution of 4000 $\times 2642$ pixels was used to record two-dimensional particle images in the central plane of the rotor wake. The flow was seeded with $10 \mu \mathrm{m}$ silver-coated glass particles and illuminated using a Nd:YAG pulsed laser (Quantel Twins Ultra 190), positioned below the shaft axis. Measurements were performed for 19 different phases $\phi$ of the blade position. 60 images were recorded for each phase and averaged for further evaluation. The PIV image analysis and subsequent post-processing were performed with in-house MATLAB codes (see Meunier and Leweke [38]). The final correlation window size was $32 \times 32$ pixels, with an overlap of $50 \%$. The resulting spatial resolution was adequate for the overall survey of the velocity field, but not sufficient for a valid determination of the vortex core characteristics (only 2 vectors inside the vortex core expected for this configuration, see also Fig. 17). A median filter with a 0.1 pixel threshold was used to detect and replace outliers and spurious vectors.

\subsubsection{ILR experiment}

The experiment at ILR is scaled to be 3 times bigger than the one at IRPHE, to ensure a sufficiently large core size of the tip vortices for resolved velocity measurements in the core region. The rotor blade is mounted at the end of a shaft of diameter $6 \mathrm{~cm}$, in the centre of a water channel test section of dimensions $1 \mathrm{~m} \times 1.5 \mathrm{~m} \times 6.5 \mathrm{~m}$. The positions of both the rotor and the laser for PIV velocity measurements (see below) are controllable by a variety of linear axes.

Dye visualisations were carried out to examine the spatial evolution and interactions of the initial vortex system. Two internal channels in both blade and fin were used to inject the dye into the flow. The rotor was designed with a hollow shaft to allow the dye feed through internal tubes. A controllable linear axis was used to adjust the volumetric flow rate to the rotational speed of the rotor. Images were recorded at a frame rate of $70 \mathrm{~Hz}$ using a 12 megapixel XIMEA CB120xG-CM camera.

Stereoscopic PIV images of the rotor centre plane were recorded by two CCD cameras (PCO.2000) with a resolution of $2048 \times 2048$ pixels. Following the recommendation of Lawson and $\mathrm{Wu}$ [39], both cameras had a collective angle of approximately $30^{\circ}$. To prevent aberrations due to the change of fluids in the optical path, water-filled prisms were used, as suggested by Prasad and Jensen [40] for optical measurement techniques in water channels. The flow was illuminated by a double-pulsed laser (Quantel Twins Ultra) providing a pulse energy of $120 \mathrm{~mJ}$ operating at an effective wavelength of $532 \mathrm{~nm}$. Cubic polyamide particles of mean diameter $50 \mu \mathrm{m}$ and density $1.016 \mathrm{~g} / \mathrm{cm}^{3}$ were used to seed the flow. To prevent peak-locking errors, the foci were adjusted to secure a mean particle diameter of 3 pixels in the recorded images [41]. The recordings for each plane were synchronised with the rotor frequency via an optical encoder, combined with a micro-controller to ensure exact triggering of the cameras and laser. Both the entire rotor wake and a close-up field of view containing the vortex interaction with high spatial resolution were investigated with stereoPIV. In a first step, a wide field of view (FOV A), mapping a $30 \mathrm{~cm} \times 22 \mathrm{~cm}$ region of the flow was used to evaluate the spatial evolution of the vortex core positions. For a higher resolution, a second field of view (FOV B) with an edge length of $8 \mathrm{~cm}$ was captured. In Fig. 5, the positions of both fields of view relative to the rotor are displayed. They were 


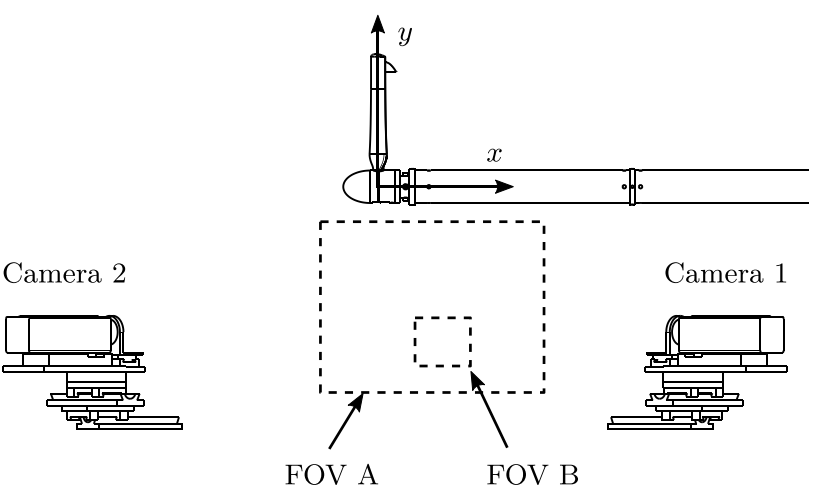

Fig. 5 Coordinate system and fields of view (FOV) for the PIV measurements in the ILR experiment

processed using a final correlation window size of $48 \times 48$ pixels, with a $75 \%$ overlap, providing 2 vectors $/ \mathrm{mm}$ for FOV $\mathrm{B}$, which yields a sufficient resolution of 8-10 vectors within the radius of the smallest vortex cores measured in these experiments. No additional filter for false-vector detection and replacement was applied. For the analyses presented in this paper, only the two velocity components lying in the plane of measurement were used.

For calibration, image acquisition, synchronisation of light source and cameras, as well as for image processing, the DaVis 8.4 software developed by LaVision was used. The light sheet was positioned directly below the axis of the rotor shaft. Using the spatial information from FOV A, the rotor was adjusted in its $x$ - and $y$-positions, so that the vortices were always well-centred in the frame. Phase-averaged measurements using 250 frames were performed in $18^{\circ}$ steps of the rotor angle $\phi$ (wake age) to give an insight into the temporal and spatial evolution of the interaction process.

In the following, the discussion of the observed phenomena is mostly based on data obtained from phase-averaged velocity fields: vortex velocity profiles (core radius, maximum swirl velocity) and circulation. In both experiments, measurements were carried out in a plane containing the rotor axis. The flow in this plane is primarily contained within the plane; out of plane velocities appear only in the vortex cores. Precise vortex characteristics were only deduced from the stereo-PIV measurements, where any perspective distortion errors are taken care of through calibration. Instantaneous PIV velocity measurements have an expected uncertainty of 2-3\%, whereas phase-averaged velocities converge to within well below $1 \%$. Based on the observed variability, the circulation uncertainty can be estimated as $5-7 \%$. The determination of vortex core radii and maximum swirl velocities is affected by the phenomenon of vortex wandering (see [42] and Sect. 4.2). The resulting uncertainty for these parameters is of the order of $5-10 \%$.

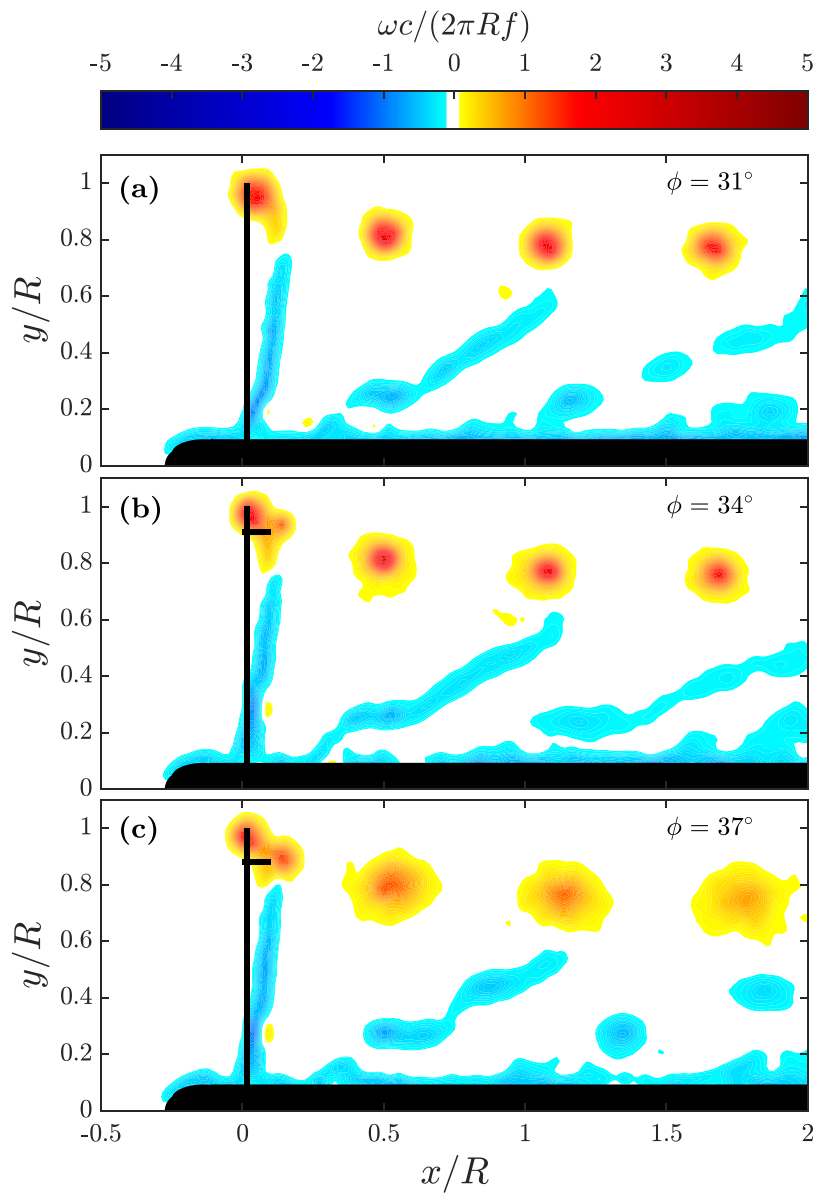

Fig. 6 Phase-averaged vorticity in the centre plane of the small-scale rotor. Blade without fin (a), and with fin at angle $\alpha_{\mathrm{f}}=6^{\circ}(\mathbf{b})$ and $\alpha_{\mathrm{f}}=14^{\circ}(\mathbf{c})$

\section{Results and discussion}

\subsection{Small-scale experiment (IRPHE)}

In this section, the results obtained from the small-scale experiments performed at IRPHE are presented. The fin parameters are $c_{\mathrm{f}} / c=0.8, d / c=0.2, h / c=0.3$ for the configurations discussed below, and the fin angle is varied in the range $6^{\circ} \leq \alpha_{\mathrm{f}} \leq 18^{\circ}$. A qualitative impression of the wake generated by the different blade configurations is given by the phase-averaged vorticity fields shown in Fig. 6, where the black lines represent the trailing edges of the rotor and the fin. The reference case without fin is shown in Fig. 6a, exhibiting the characteristic wake development of a rotating blade, with a concentrated helical tip vortex, which traverses the centre plane of the rotor at regular intervals, and a vorticity sheet of opposite sign in the inner part. In comparison, the wakes generated by the modified rotor blades show remarkable differences, displayed in Fig. 6b, c. The additional fin indeed generates a secondary vortex which 
interacts with the primary tip vortex. After one rotor revolution, the secondary vortex cannot be detected anymore, implying that a vortex merging process has taken place, which has a significant effect on the final vortex characteristics. This is particularly visible in Fig. 6c. The strength of the secondary vortex, and consequently the circulation ratio, is affected by the fin angle $\alpha_{\mathrm{f}}$.

As anticipated from theoretical considerations, a pair of vortices of approximately equal strength is favorable for the desired result. The fin vortex in Fig. $6 \mathrm{~b}\left(\alpha_{\mathrm{f}}=6^{\circ}\right)$, has a noticeably weaker effect than the one in Fig. $6 \mathrm{c}\left(\alpha_{\mathrm{f}}=14^{\circ}\right)$. Merging processes involving vortices of unequal strengths have been analysed in the work of Dritschel and Waugh [43] and Brandt and Nomura [44]. The asymmetry leads to a stretching and ultimate collapse of the weaker vortex in the strain field generated by the stronger vortex. In comparison to a complete merging process, the effects on the core radius and the tangential velocities of the remaining vortex are much smaller.

To evaluate the strength ratio of the vortex pair and the final vortex, the circulation is obtained from suitably chosen line integrals of the velocity around vortices (see Sect. 4.2 below). The unmodified blade is used as a reference, and the circulation of each distinct vortex, along with the total circulation, is calculated for all blades. The results for the cases shown in Fig. 6 are depicted in Fig. 7. Increasing the fin angle $\alpha_{\mathrm{f}}$ results in a stronger fin vortex, while the circulation of the tip vortex is slightly decreasing. Fin-to-tip circulation ratios $\left(\Gamma_{\mathrm{f}} / \Gamma_{\mathrm{t}}\right)$ close to one, favourable for a complete merging, are achieved with higher fin angles. The time scale of the merging process is also influenced, it increases as the circulation ratio, gets closer to the desired value of 1 . In the case of Fig. $7 \mathrm{~b}$, the merging starts at a wake age of $\phi \approx 200^{\circ}$. From this point on, the distinct vortex centres cannot be detected anymore. For unequal vortex pairs (Fig. 7a), the deformation and straining of the weaker vortex is much faster, but the effects on the final vortex are hardly visible. For all investigated cases, the merging process was completed at the latest at $\phi \approx 290^{\circ}$.

It should be noted that the total circulation of the tip vortex system generated by the rotor blades equipped with a fin is always higher than the the one of the unmodified blade, which indicates an increase in total lift. The presence of the fin has also an effect on the rotor performance (thrust, power). A first estimate of this influence is given in Sect. 4.3 below.

The influence of the fin angle $\alpha_{\mathrm{f}}$ on the total circulation and the circulation ratio $\Gamma_{\mathrm{f}} / \Gamma_{\mathrm{t}}$ can be seen in Fig. 8. As mentioned before, the total circulation $\Gamma_{\text {tot }}$ is larger than the reference value $\Gamma_{0}$ for cases with fin, increasing almost linearly with increasing fin angle. The circulation ratio of the initial vortex pair shows a similar trend, and $\Gamma_{\mathrm{f}} / \Gamma_{\mathrm{t}}$ even exceeds 1 for the highest considered fin angle of $\alpha_{\mathrm{f}}=18^{\circ}$.

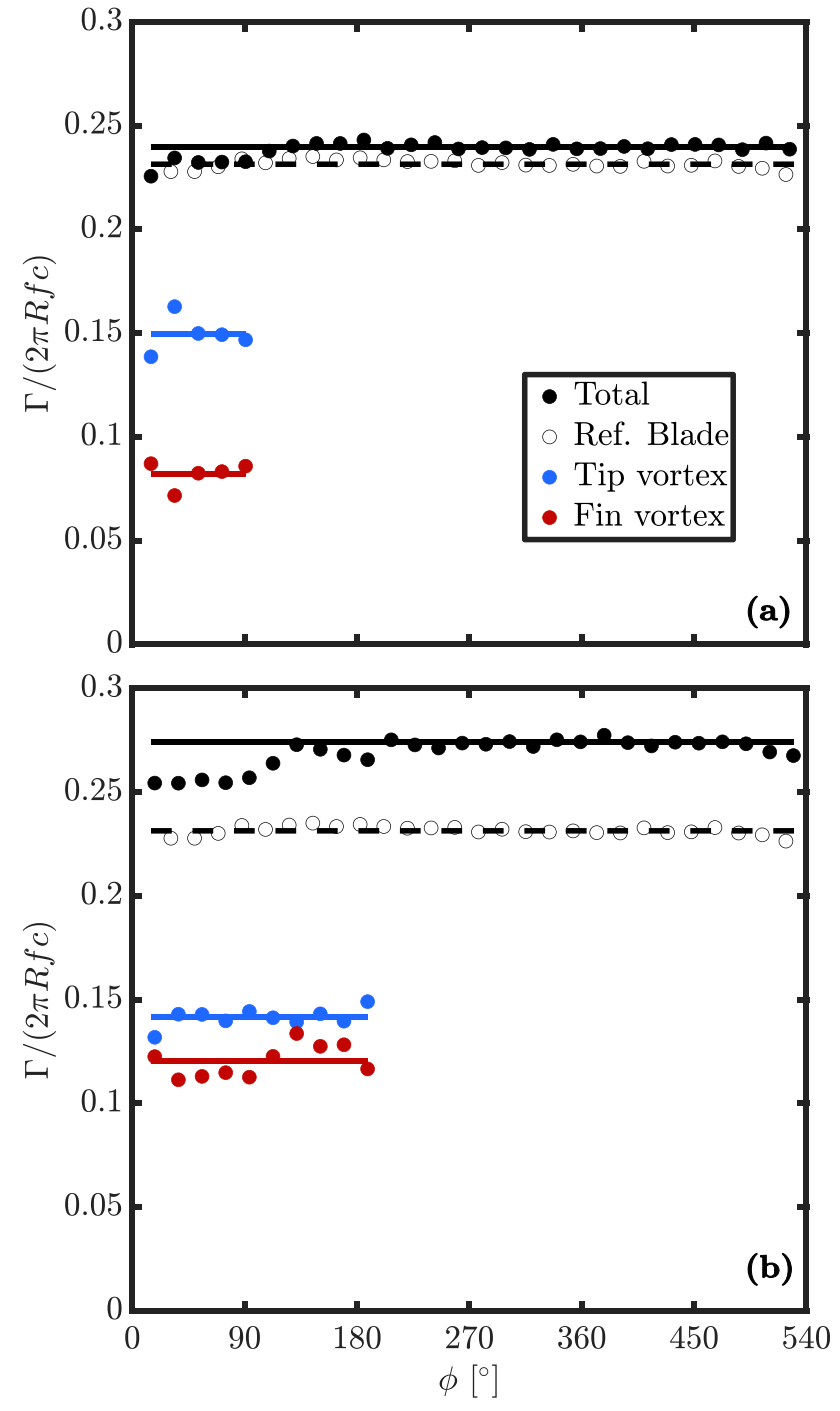

Fig. 7 Vortex circulations for the cases with fin at angle $\alpha_{\mathrm{f}}=6^{\circ}$ (a) and $\alpha_{\mathrm{f}}=14^{\circ}(\mathbf{b})$

The velocity fields measured in this series of experiments can be used to obtain a first qualitative assessment of the outcome of the merging process, as function of the circulation ratio of the initial vortex pair, which is here considered as the relevant parameter. Figure 9 depicts the observed relation between $\Gamma_{\mathrm{f}} / \Gamma_{\mathrm{t}}$ and the core radius of the merged vortex, normalised with the value for the reference blade. These radii were deduced from the velocity profiles of the vortices, using the procedure described in more detail in the following section. They represent the core sizes after about one rotor revolution. The variation is almost negligible for unequal vortex pairs prone to incomplete or partial merging. For ratios closer to $1\left(\Gamma_{\mathrm{f}} / \Gamma_{\mathrm{t}}>0.75\right)$, more likely to undergo a complete merging processes, the radius of the merged vortex is significantly larger than for the reference blade without fin. A somewhat unexpected behaviour was found for a fin 


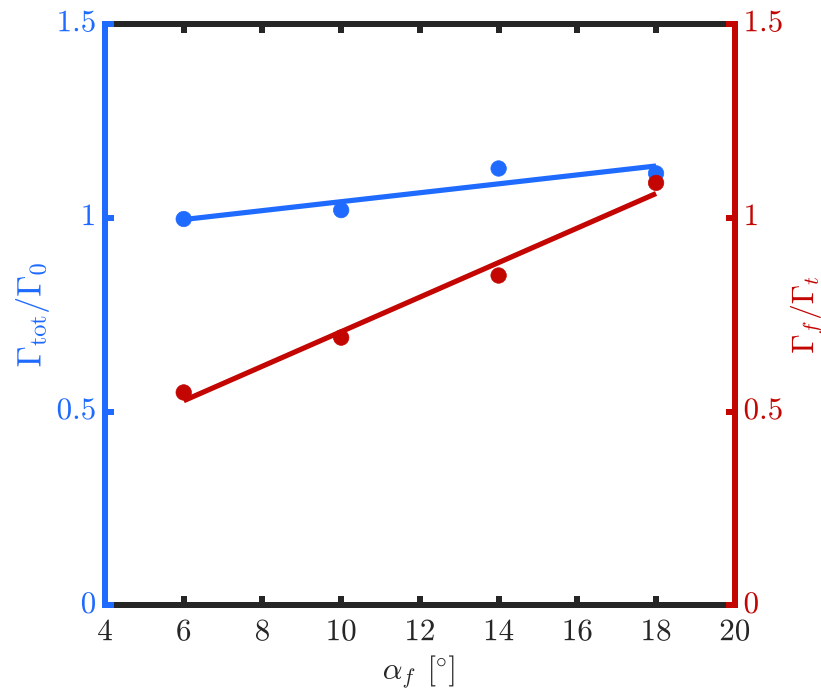

Fig. 8 Total circulation and fin-to-tip circulation ratio as function of the fin angle

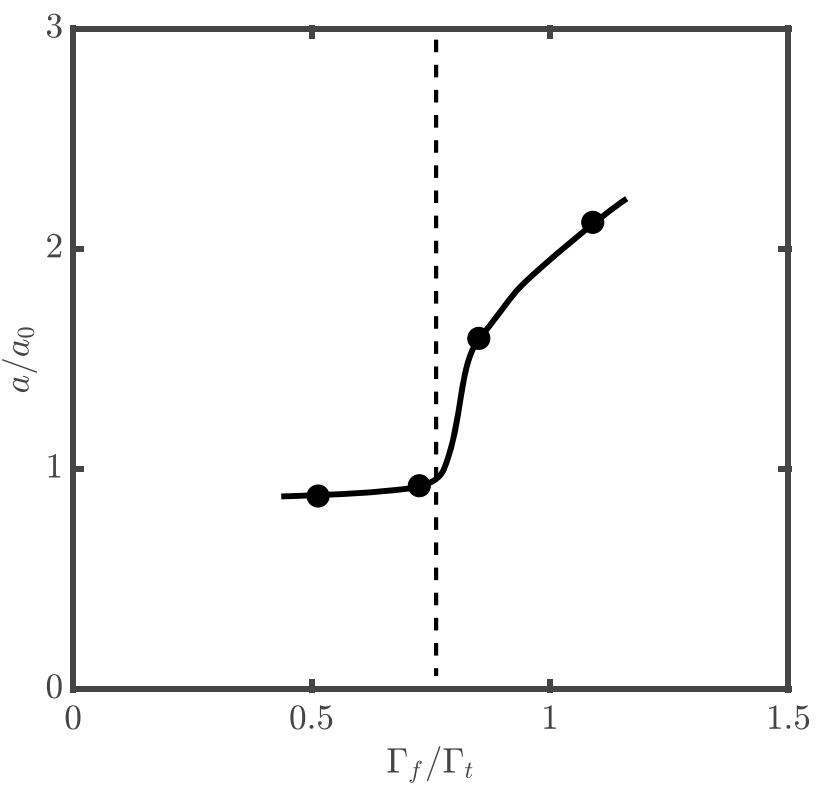

Fig. 9 Variation of the final core size (merged vortex) as function of the circulation ratio

angle of $\alpha_{\mathrm{f}}=18^{\circ}$, which generated a circulation ratio greater than 1 . The resulting core radius was even larger than for the case closest to the theoretical optimum of $\Gamma_{\mathrm{f}} / \Gamma_{\mathrm{t}}=1$.

Due to the small scale of the experiment performed at IRPHE, the velocity measurements in the regions of the vortex core lack the required spatial resolution. Thus, the results concerning the core size and its variation should be interpreted only as as qualitative estimate of the effects achieved by the modifications of the blade tip geometry. To obtain valid quantitative results with an appropriate PIV resolution and to analyse correctly the scalability of the blade design, the measurements from the larger-scale ILR experiment have to be considered.

\subsection{Larger-scale experiment (ILR)}

The IRPHE experiment has shown that the generation of a tip vortex pair using a fin is also possible for the case of a rotating blade, and it permitted to identify configurations for which the desired core size increase is significant. In this section we focus on one of these configurations, which produces a pair of co-rotating tip vortices whose circulation ratio is close to one, to obtain more precise information about the core evolution. The fin parameters for this case are: $c_{\mathrm{f}} / c=0.8, d / c=0.2, h / c=0.3$ and $\alpha_{\mathrm{f}}=14^{\circ}$.

A qualitative analysis of the vortex structure can be obtained from dye visualisations. Information about the initial position and spatial evolution of the vortex system provides valuable information for the subsequent PIV measurements. Figure 10a shows the helical vortex generated by the unmodified blade. The formation of a concentrated, stable tip vortex is visible, its core dimensions seem to grow slightly after two revolutions. Considering the core evolution, a disturbance affecting the overall helix geometry is visible in the later stages. The low free-stream velocity used in this case is affected by non-uniformities over the rotor diameter. Parts of the vortex are transported with slightly higher velocities, causing the visible deformation of the helix. This disturbance is not related to any long-wavelength instability phenomenon.

In comparison to the unmodified blade, the rotor wake of the modified blade shows clear differences. The corresponding visualisation obtained with the same flow parameters is displayed in Fig. 10b. In addition to the primary vortex generated at the blade tip, a secondary vortex emerges from the fin tip. This secondary vortex appears to be less stable than the primary one, indicated by the disturbed dye filaments in the core region. After one revolution, the two initial vortices have merged into a single one. Visually, the core of the final vortex appears to be strongly widened, compared to the unmodified case in Fig. 10a. Due to the rapid diffusion of the dye, a quantitative analysis of the core dimensions is not feasible. Nevertheless, these images clearly show the effect of the tip modification concerning the formation of two distinct vortices, and indicate that a strong and rapid vortex interaction takes place.

The PIV analysis provides detailed quantitative information supporting the visual impressions. The measured velocity fields were used to determine the base flow properties of the rotor wake. In the close-up field of view B (see Fig. 5), the vortex evolution is recorded over one rotor revolution in steps of $18^{\circ}$ in the rotor phase $\phi$. In Fig. 11a, the phase-averaged vorticity in a plane containing the rotor 

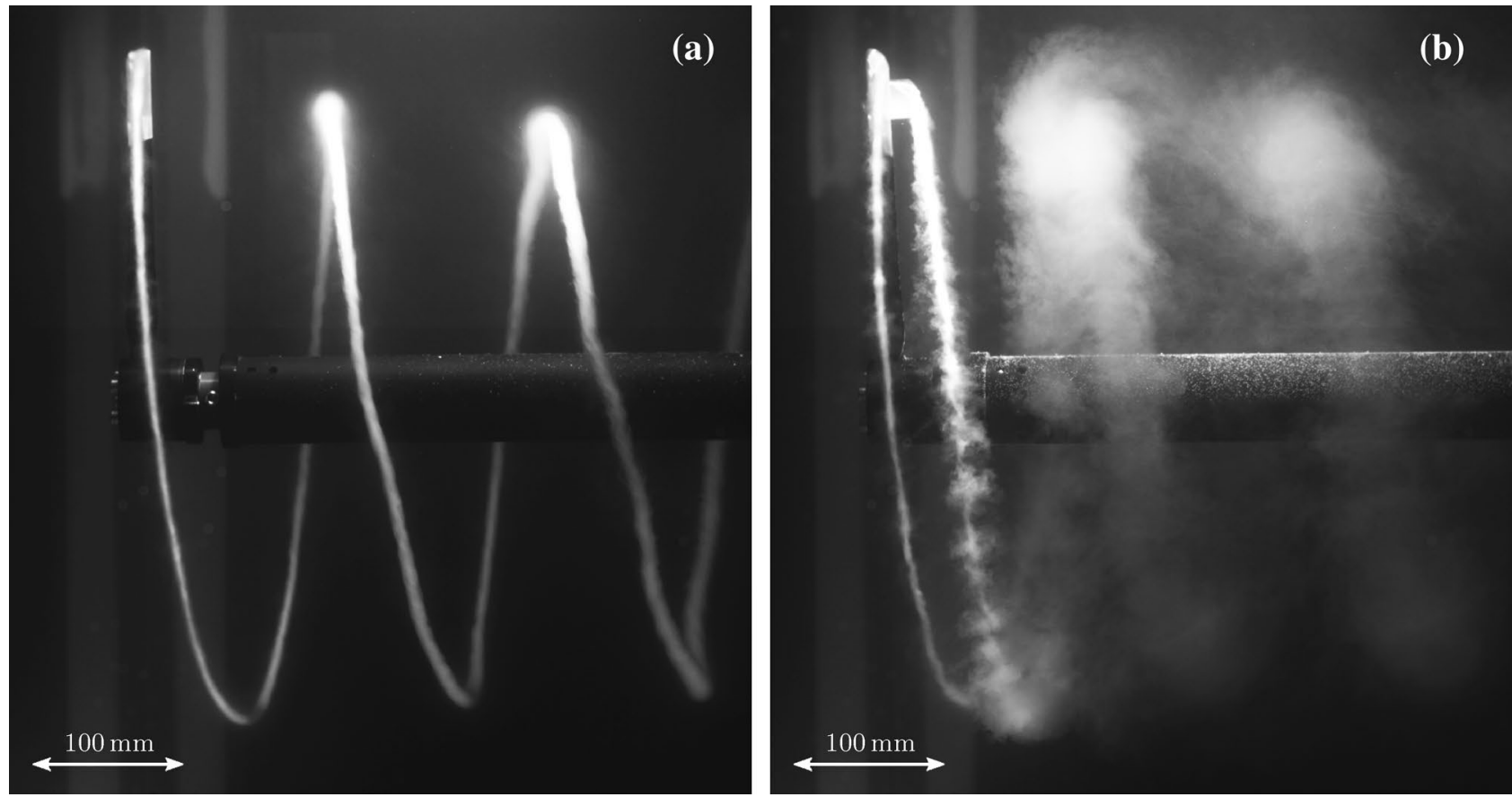

Fig. 10 Dye visualisations of the rotor wake for the cases without (a) and with (b) blade tip fin. Flow is from left to right

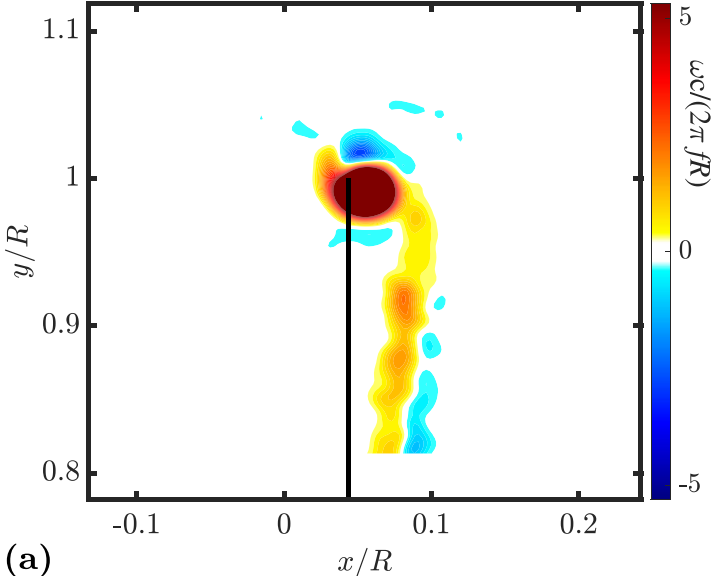

Fig. 11 Phase-averaged vorticity in the centre plane of the rotor for the unmodified (a) and modified (b) blade at $\phi=18^{\circ}$. The black lines represent the positions of the trailing edges of the blade and fin at

axis, obtained from 250 instantaneous fields, is plotted for the unmodified rotor at $\phi=18^{\circ}$. The single, concentrated vortex emerging from the blade tip is clearly visible, the roll-up process is completed. This represents the reference case. Regarding the initial vortex system, the wake generated by the modified blade differs significantly. Two distinct vortices of similar size are formed at the tip and the fin, which create a co-rotating vortex pair prone to vortex merging. The corresponding vorticity field is shown in Fig. 11b. This figure also illustrates how the circulation of

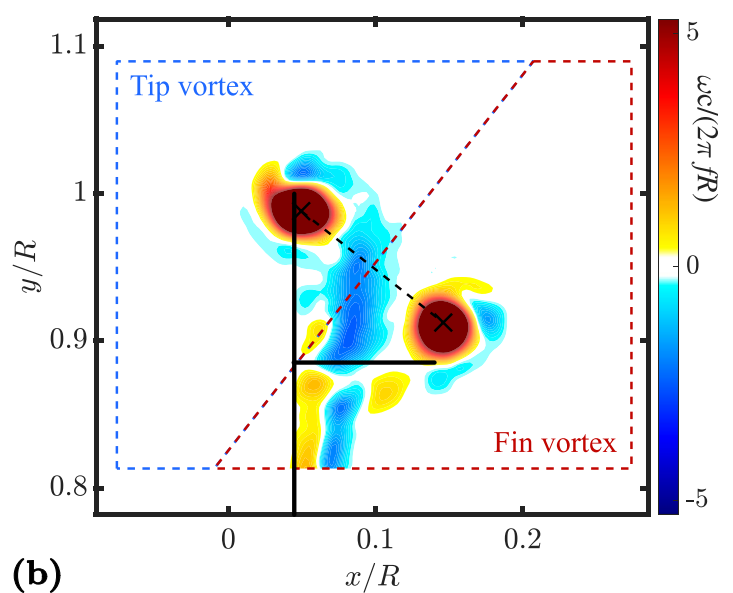

$\phi=0^{\circ}$. Blue and red polygons in $\mathbf{b}$ show the integration regions for the circulation calculation

each individual vortex was determined: the vorticity field is separated into two distinct regions, marked by the blue and red polygons. Each vortex centre is determined using the $\lambda_{2}$-criterion defined by Jeong and Hussain [45]. A separation line is drawn orthogonally to the line connecting the centres, defining the border of the line integrals for the respective region assigned to the tip or the fin vortex. Figure 12 shows the circulations calculated in this way for an entire rotor revolution. The individual quantities and the total circulation based on the entire field of view are 


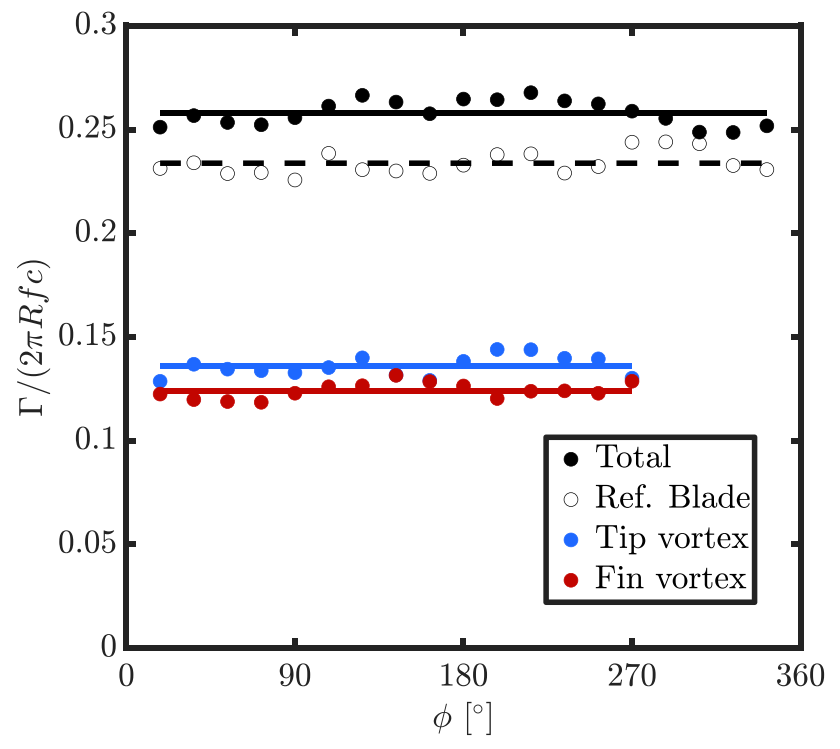

Fig. 12 Vortex circulations for the modified blade

plotted over the rotor phase $\phi$. In comparison to the reference blade, represented by the dashed line, the averaged total circulation is increased, indicating that the additional fin is enhancing the circulation generated by the blade. This is consistent with the results obtained in the IRPHE experiment. Both vortices possess almost the same circulation, as expected, making this configuration suitable for a complete merging process. The ratio of the average circulations of the two vortices is $\Gamma_{\mathrm{f}} / \Gamma_{\mathrm{t}} \approx 0.9$. At a rotor phase of $\phi=270^{\circ}$, the merging process is almost completed, and two distinct vortex centres cannot be detected anymore.

The evolution of the tip vortex pair with wake age is shown in Fig. 13, illustrated by phase-averaged vorticity fields. Meunier et al. [29] found that for two-dimensional co-rotating vortices, merging sets in when the ratio $a / b$ of the Gaussian core radius $a$ and the vortex separation distance $b$ exceeds approximately 0.24 . Below this value, one observes a slow increase of this ratio, due to the growth of the core radius on a viscous time scale (see Eq. (1)), whereas the separation distance $b$ remains essentially constant. Figure $13 \mathrm{a}-\mathrm{c}$ show the vortex system before the critical ratio is reached at a rotor phase of $\phi \approx 130^{\circ}$. Both vortices have similar circulation (Fig. 12), and rotate around each other while moving downstream. The rapid growth of the core size is qualitatively visible. Meunier et al. [29] pointed out the significant influence of the elliptic instability on the merging process. Considering the high Reynolds number at the blade tip $(R e=120,000)$, mutual instabilities affecting the growth rates of the radii might in principle occur during the initial stages of the flow, as discussed in Sect. 2.2 above. An estimate of the corresponding growth rates, based on the initial vortex pair characteristics and available theory suggests that it would not be high enough to explain the observed rapid destabilisation.

A closer analysis of the circulation profiles of the tip and fin vortices hints at the possibility of a centrifugal instability, related to the presence of opposite-signed vorticity, which is visible in Figs. 11b and 13a. This aspect has been analysed in more detail elsewhere [46, 47]. The presence of this instability accelerates the core growth and in consequence the onset of the merging process. Figure $13 \mathrm{~d}-\mathrm{f}$ show vorticity fields during merging. A deformation of the vortex structure combined with a decrease of the core separation occurs, resulting in the combination of both vortices into a single one. During this process, the vortex centres and core radii cannot be accurately detected in the experimental measurements. After completion of merging (at $\phi \approx 290^{\circ}$ ), the final vortex exhibits a strongly widened core radius compared to the initial state. From this point onwards, only a slow growth is observed, which is in accordance with theoretical predictions for the viscous diffusion of vorticity.

The velocity fields obtained from the PIV measurements allow a detailed analysis to further extract the relevant vortex parameters. After identifying the vortex centres, the distance between the vortices can be determined. A projection of the velocities onto a local polar grid $(\rho, \varphi)$ is made to calculate the radial profile of the (average) swirl velocity $v$; the maximum of this profile is used to determine the core radius: $v_{\max }=v\left(\rho=a_{\max }\right)$. It was shown, e.g. in Ref. [42], that the phenomenon of vortex wandering could lead to errors in the determination of core velocity profiles (radius, maximum velocity) from phase-averaged fields. Estimates based on the results in [42] show that these effects are small compared to those induced by the fin. They were therefore neglected in the present analysis.

In Fig. 14a, these profiles are plotted for the modified and the reference cases at a rotor phase $\phi=18^{\circ}$, the dashed line indicating the core radius for the reference case. Tip and fin vortex exhibit an almost identical structure. After the merging process is completed, the peak tangential velocity is considerably reduced, and the core size is much larger, compared to the reference case (Fig. 14b). The evolution of the maximum tangential velocity for the tip and fin vortices for all measured rotor phases $\phi$ is plotted in Fig. 15. An abrupt decrease is notable in both vortices, indicating an immediate interaction between them. The maxima decrease down to $25 \%$ of their initial values at a rotor phase of $\phi \approx 180^{\circ}$. Once the vortices have merged, the peak velocity of the final vortex remains essentially constant. The single vortex from the unmodified blade shows only a slight decrease during the first rotor period. After one revolution, the peak velocity of the merged vortex is about 3.5 times lower than for the reference case. A visual illustration of the differences between both final vortices is given by the vorticity fields in Fig. 16, emphasising the effect of the fin on the rotor wake. 


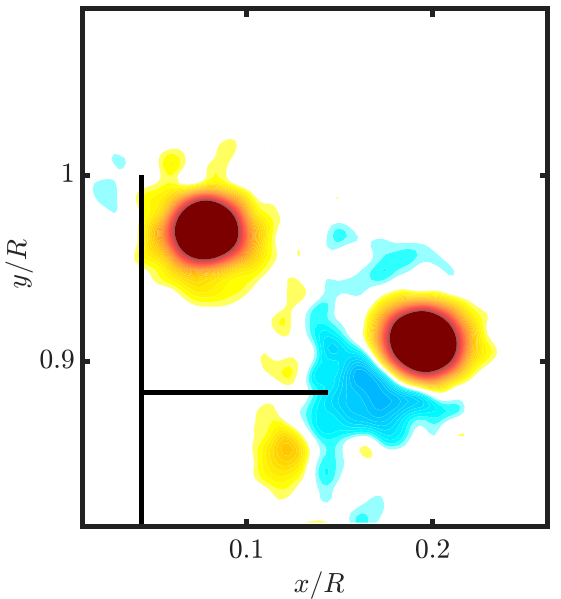

(a) $\phi=36^{\circ}$

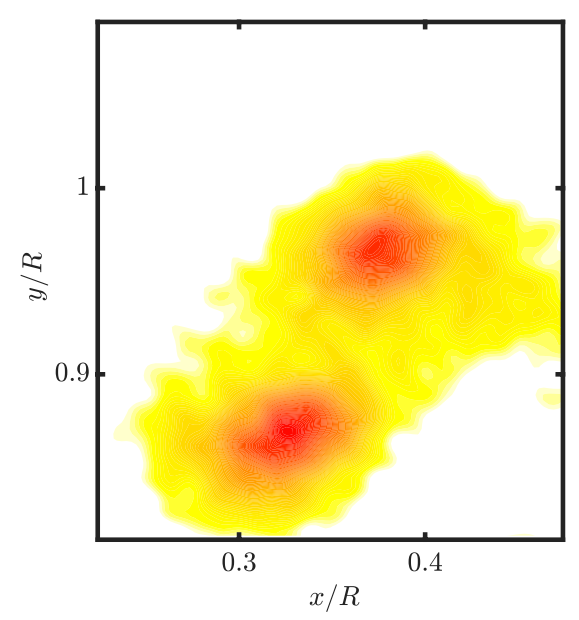

(d) $\phi=144^{\circ}$

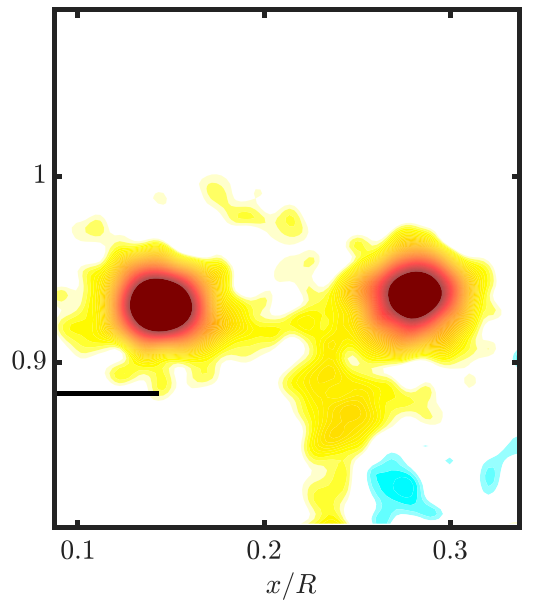

(b) $\phi=72^{\circ}$

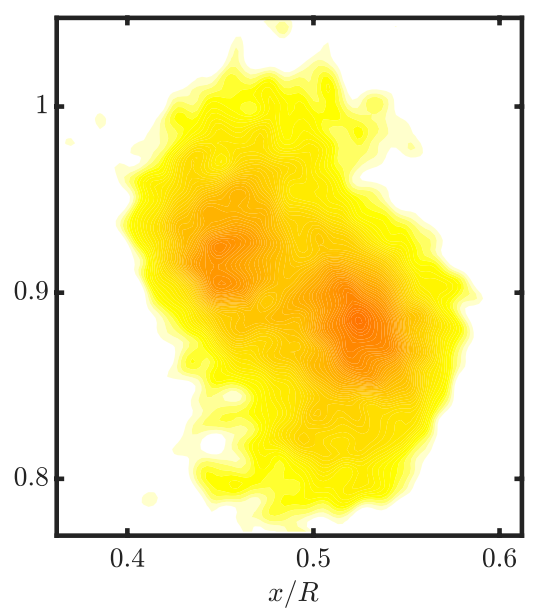

(e) $\phi=216^{\circ}$

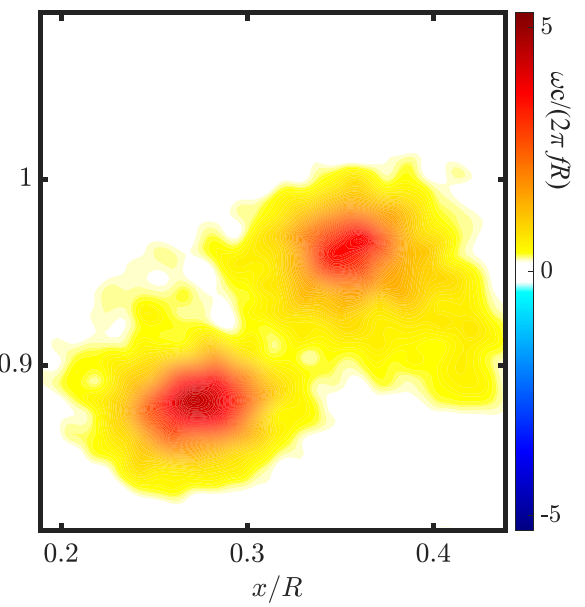

(c) $\phi=126^{\circ}$

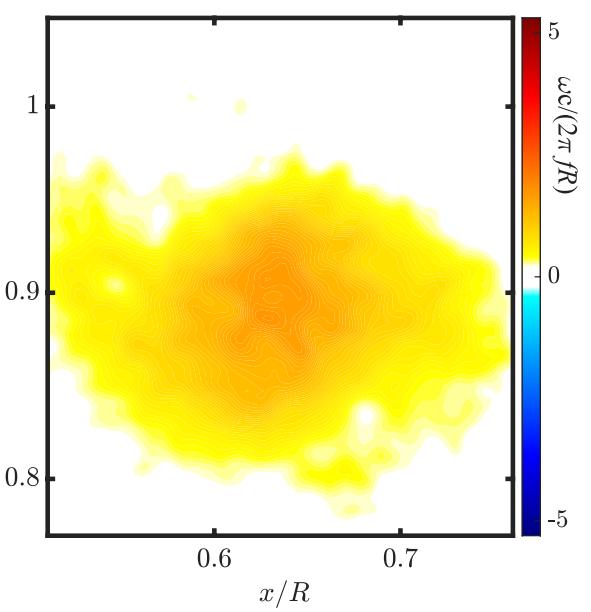

(f) $\phi=288^{\circ}$

Fig. 13 Phase-averaged vorticity fields for different rotor phases. The black lines represent the trailing edges of the blade and the fin at $\phi=0^{\circ}$
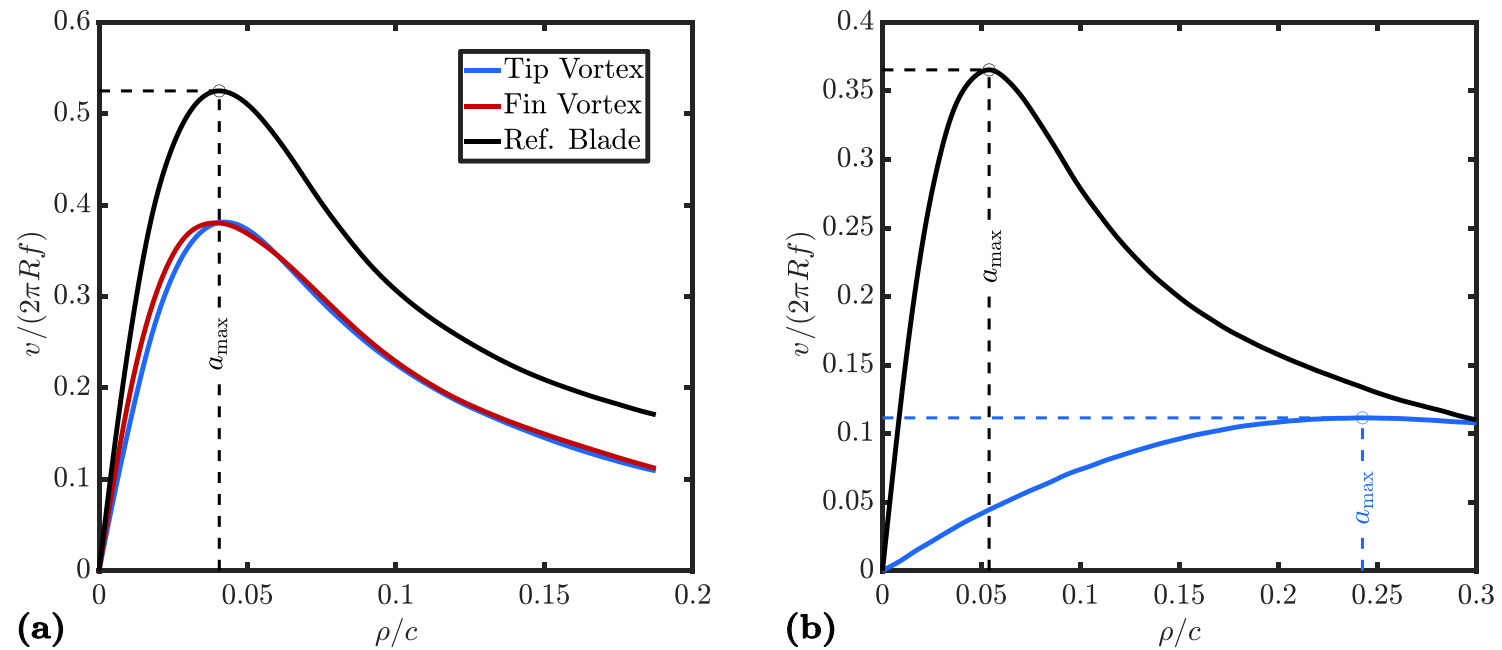

Fig. 14 Swirl velocity profiles for the modified blade and for the reference case at $\phi=18^{\circ}(\mathbf{a})$ and $\phi=342^{\circ}(\mathbf{b})$. The dashed lines indicate the core radii $a_{\max }$ 


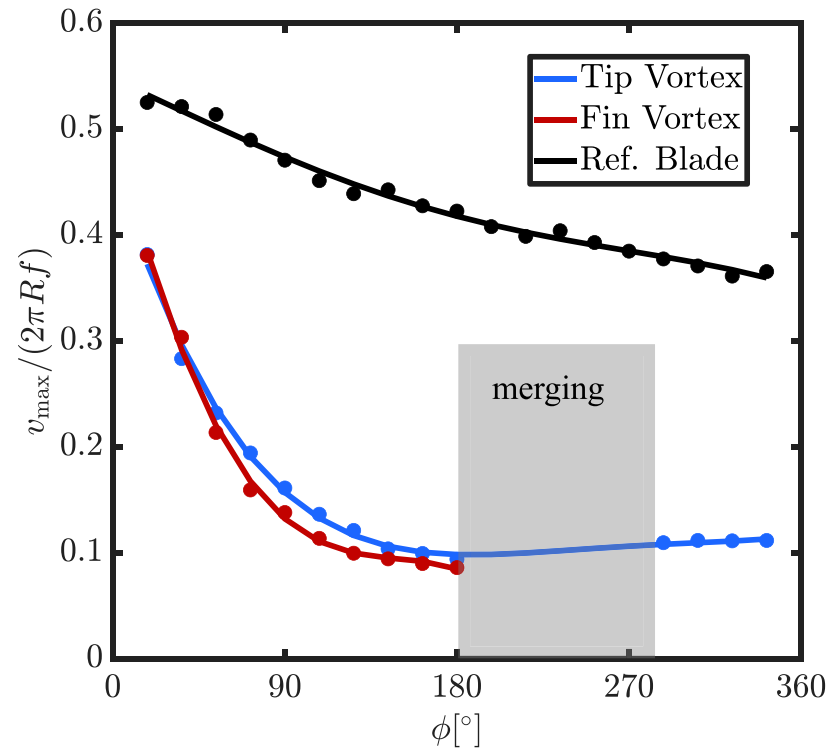

Fig. 15 Evolution of the maximum swirl velocities over one rotor revolution. The grey region comprises the merging process

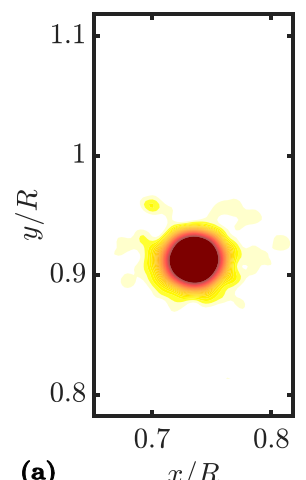

(a)

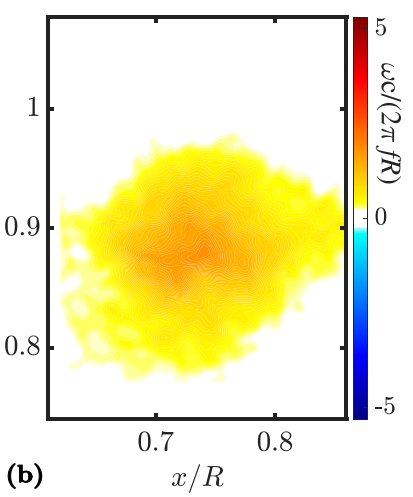

Fig. 16 Phase-averaged vorticity fields of the (merged) tip vortex after one blade revolution. $\mathbf{a}$ Without and $\mathbf{b}$ with fin

Regarding the evolution of the vortex core radius in unperturbed laminar flow, the growth is dominated by a viscous diffusion law (Eq. (1)). In Fig. 17, the evolution of the normalised Gaussian core radius with the rotor phase is plotted. Comparison between the predicted growth rate and the experimental data shows good agreement. This indicates that, despite the fairly high Reynolds number, the inner core region appears to be laminar.

The development of the core radii in the modified case is displayed in Fig. 18, which shows strong differences to Fig. 17. Both vortices exhibit an immediate rapid growth of their core radii, visible from $\phi=18^{\circ}$ up to $\phi=180^{\circ}$. The conglomeration of the vortices, starting at approximately $\phi=180^{\circ}$ inhibits a valid detection of the core radii, since a precise peak in the tangential velocity cannot be

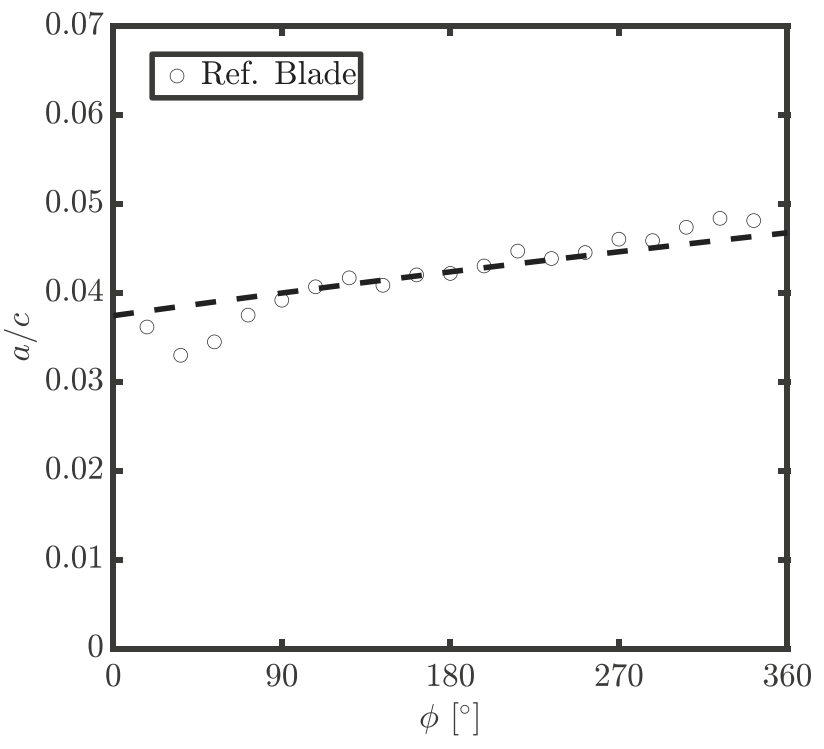

Fig. 17 Core radius evolution for the tip vortex of the unmodified blade, dashed line represents the theoretical growth rate

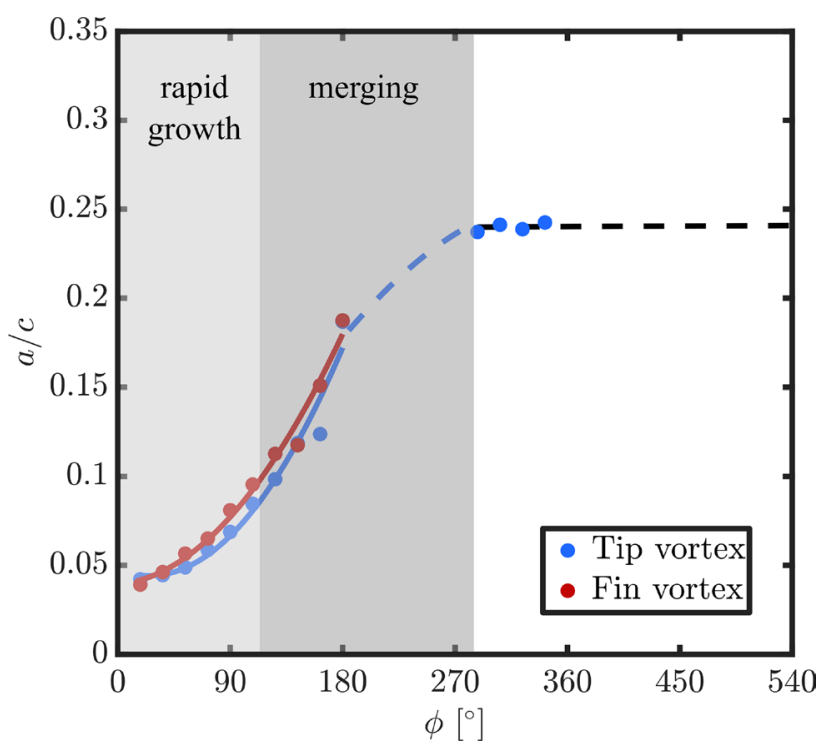

Fig. 18 Core radii evolution leading to the eventual vortex merging for the modified blade

detected anymore. This explains the missing data in the merging region in Fig. 18. After completion of the merging process (at $\phi \approx 290^{\circ}$ ), the final vortex has a normalised core radius $a / c \approx 0.245$. From this point onwards, the growth follows again the viscous diffusion law in Eq. (1). In relation to the initial state, the core size is multiplied by a factor of about 5 . One may note that for the reference blade without fin, the tip vortex would reach a comparable radius only at a wake age of roughly 50 rotor revolutions. 
The development of the ratio $a / b$ characterising the onset of merging is shown in Fig. 19. The rapid increase observed well before reaching the critical value $a / b=0.24$ indicates again the presence of a perturbation or instability having a significant influence on the growth of the core radii.

Whereas the presence of the tip fin strongly modifies the core size, the vorticity and the swirl velocities of the rotor wake vortices, the large-scale spatial structure of the vortex system is nearly unaffected. The positions of the vortex centres after one blade rotation are almost identical for both cases.

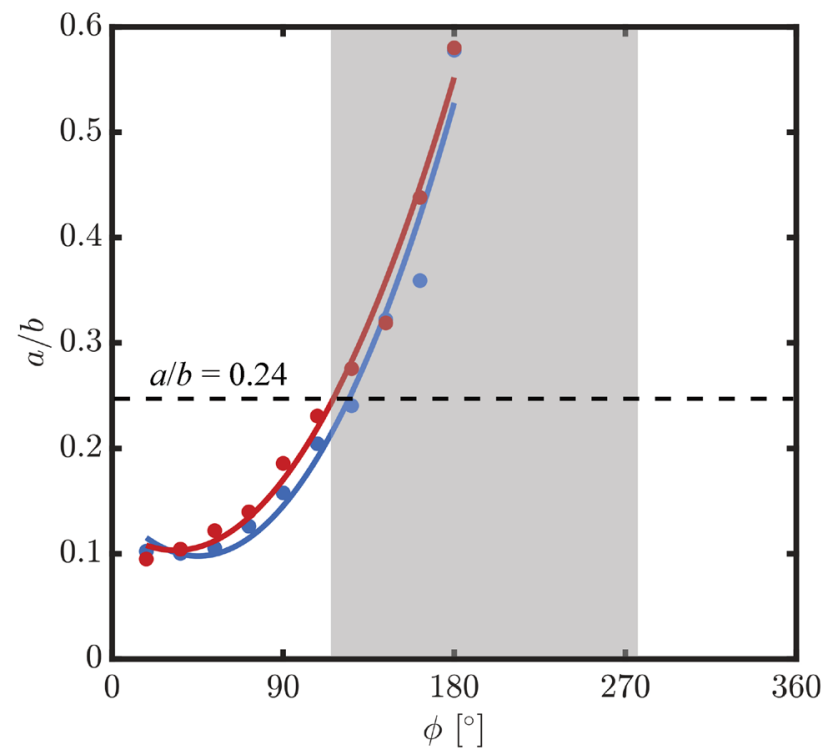

Fig. 19 Evolution of the ratio between Gaussian core radii and core separation distance
The above results relate to a single promising fin configuration. Variations of the fin parameters, such as its angle $\alpha_{\mathrm{f}}$ and position $d$ (see Fig. 3c), were also carried out in the ILR set-up, confirming the qualitative observations made in the small-scale IRPHE experiments. Some of these further results can be found in Ref. [46].

\subsection{Effects on rotor performance}

The presented modification of the blade tip geometry showed interesting results with regard to changing the characteristics of the rotor wake vortices. However, besides generating a secondary tip vortex, the additional fin is also expected to have some effect on the performance of the rotor. Force measurements were not carried out in the present experiments, but some indications can be obtained from the measured wake velocity fields. As shown above (Figs. 7, 8, 12), the total circulation generated by the modified blade is increased with respect to the reference case, which indicates that the aerodynamic characteristics of the blade are affected by the presence of the fin. The measured velocity field at $\phi=18^{\circ}$ for the ILR rotor was used to deduce the radial distribution of bound circulation on the blades; the result is displayed in Fig. 20a. It is notable that the fin has an impact not only on the maximum value close to the tip region, but also on the distribution in the inner region of the blade: the entire curve is raised, in comparison to the unmodified case. Concerning rotor performance, this higher overall circulation profile may indicate a modification of the generated thrust and the necessary power. To further evaluate this aspect, we analysed the time-averaged velocity fields downstream of the rotor in the IRPHE experiment. Figure 20b shows the profiles of mean induced axial velocity at $x / R=2.44$ for the reference case and the modified blade. Over most of
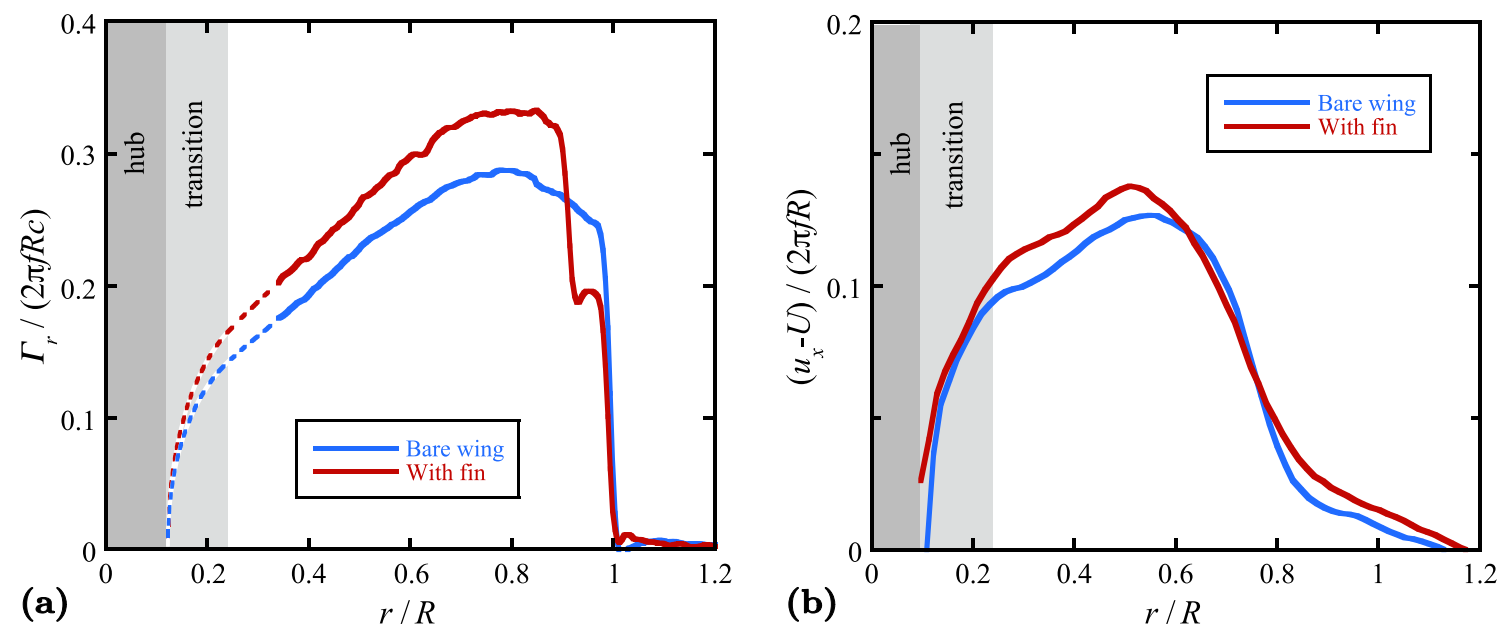

Fig. 20 Effect of the blade tip fin on the rotor performance. a Radial distributions of bound circulation for the ILR rotor $(U=5.7 \mathrm{~cm} / \mathrm{s}$ in this case). b Profiles of mean induced axial velocity at $x / R=2.44$ for the IRPHE rotor 
the wake cross-section, the induced velocity is higher in the presence of the fin. In idealised hover conditions, the rotor thrust and power are proportional to the square and the cube of the induced velocity, respectively. Using these relations, one can estimate that the addition of the tip fin in its optimal configuration results in a thrust increase of roughly $10-15 \%$, with a corresponding power increase of roughly $15-20 \%$. Given the relation between fin angle and total circulation (Fig. 20), these values are expected to increase for larger fin angles. These estimates do not take into account viscous losses and the additional drag caused by the the presence of the fin. Furthermore, the lift vector generated by the fin has a component in the direction opposite to the blade motion, causing an additional induced drag. These effects further increase the power consumption. More experimental and numerical investigations, including direct force measurements, are planned to provide more detailed information on the impact of the fin on the rotor performance characteristics. One may note that in situations where a constant thrust (or power) is required, such as helicopter flight, it is possible to balance the increase induced by the fin by lowering the collective pitch of the main blades.

\section{Conclusion}

We have presented the results of an experimental study of two closely spaced helical vortices, created by a generic rotor blade equipped with a parametric fin. The objective was to modify the properties of the rotor wake vortex system, to reduce negative effects of blade-vortex interactions that may appear in certain regimes of rotor operation. Splitting the single concentrated blade-tip vortex into two separate co-rotating vortices was expected to produce a final merged vortex with a larger core size and lower gradients, which reduces the strength of these interactions. The present study has shown that this goal can be achieved with the proposed new blade tip geometry. When a perpendicular fin is placed on the pressure side of the blade, a strong secondary vortex appears. A series of water channel experiments with a small-scale single-bladed rotor confirmed that this method, first developed for a fixed wing, is also suitable for rotors. Variation of the fin position and geometry allows to change the vortex parameters, and in particular to produce pairs of vortices having almost the same circulation. Details of the evolution of such a tip vortex pair were obtained by further experiments at a larger scale, which included dye visualisations and velocity measurements. They revealed that the merging of the co-rotating vortices proceeds surprisingly fast, indicating the presence of an instability causing an early rapid core growth in this configurations. The merged vortex after one rotor rotation is found to be 5 times larger than the one shed without a fin, with a significantly reduced peak tangential velocity, which means that the initial objective of this study was achieved. Since the relevant scale for the evolution of the tip vortex system is the blade chord, one can expect that the merging will occur at a much lower wake age for blades with higher aspect ratio, e.g. those of helicopter rotors, and that the presented blade modification could therefore have potential for reducing blade-vortex interaction noise.

Further work includes a systematic parameter study concerning the variation of the optimal fin geometry as function of the rotor characteristics (tip speed ratio, thrust), as well as a thorough investigation of the instability mechanism responsible for the rapid initial core growth. For this, volumetric PIV measurements and supporting numerical calculations are also planned. Additional force and power measurements to further analyse the influence of the added fin on rotor performance are intended.

Acknowledgements This work is part of the German-French project TWIN-HELIX, supported by the Deutsche Forschungsgemeinschaft (Grant no. 391677260) and the French Agence Nationale de la Recherche (Grant no. ANR-17-CE06-0018).

Funding Open Access funding enabled and organized by Projekt DEAL.

Open Access This article is licensed under a Creative Commons Attribution 4.0 International License, which permits use, sharing, adaptation, distribution and reproduction in any medium or format, as long as you give appropriate credit to the original author(s) and the source, provide a link to the Creative Commons licence, and indicate if changes were made. The images or other third party material in this article are included in the article's Creative Commons licence, unless indicated otherwise in a credit line to the material. If material is not included in the article's Creative Commons licence and your intended use is not permitted by statutory regulation or exceeds the permitted use, you will need to obtain permission directly from the copyright holder. To view a copy of this licence, visit http://creativecommons.org/licenses/by/4.0/.

\section{References}

1. Yu, Y.H.: Rotor blade-vortex interaction noise. Prog. Aerosp. Sci. 36, 97-115 (2000)

2. Zhao, R., Shen, W., Knudsen, T., Bak, T.: Fatigue distribution optimization for offshore wind farms using intelligent agent control. Wind Energy 15, 927-944 (2012)

3. Schmitz, F.H., Yu, Y.H.: Helicopter impulsive noise: theoretical and experimental status. J. Sound Vib. 109, 361-422 (1986)

4. Yu, Y.H., Gmelin, B., Splettstoesser, W., Philippe, J.J., Prieur, J., Brooks, T.F.: Reduction of helicopter blade-vortex interaction noise by active rotor control technology. Prog. Aerosp. Sci. 33, 647-687 (1997)

5. Brocklehurst, A., Barakos, G.N.: A review of helicopter rotor blade tip shapes. Prog. Aerosp. Sci. 56, 35-74 (2013)

6. Schröder, D., Leweke, T., Hörnschemeyer, R., Stumpf, E.: Generation of a wingtip vortex pair using a pressure-side fin. Aerosp. Sci. Technol. (2021) (Submitted) 
7. Leweke, T., Le Dizès, S., Williamson, C.H.K.: Dynamics and instabilities of vortex pairs. Annu. Rev. Fluid Mech. 48, 507-541 (2016)

8. Leweke, T., Quaranta, H.U., Bolnot, H., Blanco-Rodríguez, F.J., Le Dizès, S.: Long- and short-wave instabilities in helical vortices. J. Phys. Conf. Ser. 524, 012154 (2014)

9. Widnall, S.E.: The stability of a helical vortex filament. J. Fluid Mech. 54, 641-663 (1972)

10. Gupta, B.P., Loewy, R.G.: Theoretical analysis of the aerodynamic stability of multiple, interdigitated helical vortices. AIAA J. 12, 1381-1387 (1974)

11. Kawada, S.: Induced velocity by helical vortices. J. Aeronaut. Sci. 1936, 86-87 (1936)

12. Hardin, J.C.: The velocity field induced by a helical vortex filament. Phys. Fluids 25, 1949 (1982)

13. Okulov, V.L.: On the stability of multiple helical vortices. J. Fluid Mech. 521, 319-342 (2004)

14. Quaranta, H.U., Bolnot, H., Leweke, T.: Long-wave instability of a helical vortex. J. Fluid Mech. 780, 687-716 (2015)

15. Quaranta, H.U., Brynjell-Rahkola, M., Leweke, T., Henningson, D.S.: Local and global pairing instabilities of two interlaced helical vortices. J. Fluid Mech. 863, 927-955 (2019)

16. Moore, D.W., Saffman, P.G.: The instability of a straight vortex filament in a strain field. Proc. R. Soc. Lond. A 346, 413-425 (1975)

17. Kerswell, R.R.: Elliptical instability. Annu. Rev. Fluid Mech. 34, 83-113 (2002)

18. Leweke, T., Williamson, C.H.K.: Cooperative elliptic instability of a vortex pair. J. Fluid Mech. 360, 85-119 (1998)

19. Meunier, P., Leweke, T.: Elliptic instability of a co-rotating vortex pair. J. Fluid Mech. 533, 125-159 (2005)

20. Roy, C., Schaeffer, N., Le Dizès, S., Thompson, M.: Stability of a pair of co-rotating vortices with axial flow. Phys. Fluids 20, 094101 (2008)

21. Roy, C., Leweke, T., Thompson, M.C., Hourigan, K.: Experiments on the elliptic instability in vortex pairs with axial core flow. J. Fluid Mech. 677, 383-416 (2011)

22. Blanco-Rodríguez, F.J., Le Dizès, S., Selçuk, C., Delbende, I., Rossi, M.: Internal structure of vortex rings and helical vortices. J. Fluid Mech. 785, 219-247 (2015)

23. Blanco-Rodríguez, F.J., Le Dizès, S.: Elliptic instability of a curved batchelor vortex. J. Fluid Mech. 804, 224-247 (2016)

24. Fukumoto, Y., Okulov, V.L.: The velocity field induced by a helical vortex tube. Phys. Fluids 17, 107101 (2005)

25. Hattori, Y., Fukumoto, Y.: Short-wave stability of a helical vortex tube: the effect of torsion on the curvature instability. Theor. Comput. Fluid Dyn. 24, 363-368 (2010)

26. Hattori, Y., Fukumoto, Y.: Modal stability analysis of a helical vortex tube with axial flow. J. Fluid Mech. 738, 222-249 (2014)

27. Blanco-Rodríguez, F.J., Le Dizès, S.: Curvature instability of a curved batchelor vortex. J. Fluid Mech. 814, 397-415 (2017)

28. Hattori, Y., Blanco-Rodríguez, F.J., Le Dizès, S.: Numerical stability analysis of a vortex ring with swirl. J. Fluid Mech. 878, 5-36 (2019)

29. Meunier, P., Le Dizès, S., Leweke, T.: Physics of vortex merging. C. R. Phys. 6, 431-450 (2005)
30. Melander, M.V., Zabusky, N.J., Mcwilliams, J.C.: Symmetric vortex merger in two dimensions: causes and conditions. J. Fluid Mech. 195, 303-340 (1988)

31. Meunier, P., Leweke, T.: Three-dimensional instability during vortex merging. Phys. Fluids 13, 2747-2750 (2001)

32. Meunier, P., Ehrenstein, U., Leweke, T., Rossi, M.: A merging criterion for two-dimensional co-rotating vortices. Phys. Fluids 14, 2757-2766 (2002)

33. Cerretelli, C., Williamson, C.H.K.: The physical mechanism for vortex merging. J. Fluid Mech. 475, 41-77 (2003)

34. Trefftz, E.: Prandtlsche Tragflächen- und Propellertheorie. Z. Angew. Math. Mech. 1, 206-218 (1921)

35. Branlard, E.: Wind Turbine Aerodynamics and Vorticity-Based Methods, Research Topics in Wind Energy, vol. 7. Springer, Cham (2017)

36. Leishman, G.J.: Principles of Helicopter Aerodynamics. CUP, Cambridge (2006)

37. Sørensen, J.N.: General Momentum Theory for Horizontal Axis Wind Turbines, Research Topics in Wind Energy, vol. 4. Springer, Cham (2016)

38. Meunier, P., Leweke, T.: Analysis and treatment of errors due to high velocity gradients in particle image velocimetry. Exp. Fluids 35, 408-421 (2003)

39. Lawson, N.J., Wu, J.: Three-dimensional particle image velocimetry: experimental error analysis of a digital angular stereoscopic system. Meas. Sci. Technol. 8, 1455-1464 (1997)

40. Prasad, A.K., Jensen, K.: Scheimpflug stereocamera for particle image velocimetry in liquid flows. Appl. Opt. 34, 7092-7099 (1995)

41. Raffel, M., Willert, C.E., Scarano, F., Kähler, C.J., Wereley, S.T., Kompenhans, J.: Particle Image Velocimetry-A Practical Guide. Springer, Cham (2018)

42. Devenporrt, W.J., Rife, M.C., Liapsis, S.I., Follin, G.J.: The structure and development of a wing-tip vortex. J. Fluid Mech. 312, 67-106 (1996)

43. Dritschel, D.G., Waugh, D.W.: Quantification of the inelastic interaction of unequal vortices in two-dimensional vortex dynamics. Phys. Fluids A 4, 1737-1744 (1992)

44. Brandt, L.K., Nomura, K.K.: Characterization of the interactions of two unequal co-rotating vortices. J. Fluid Mech. 646, 233-253 (2010)

45. Jeong, J., Hussain, F.: On the identification of a vortex. J. Fluid Mech. 285, 69-94 (1995)

46. Schröder, D., Leweke, T., Hörnschemeyer, R., Stumpf, E.: Experiments on helical vortex pairs in the wake of a rotor. In: AIAA SciTech Forum (2021). Paper 2021-1088

47. Schröder, D., Leweke, T., Hörnschemeyer, R., Stumpf, E.: Instability and merging of a helical vortex pair in the wake of a rotor. J. Phys. Conf. Ser. 1934, 012007 (2021)

Publisher's Note Springer Nature remains neutral with regard to jurisdictional claims in published maps and institutional affiliations. 